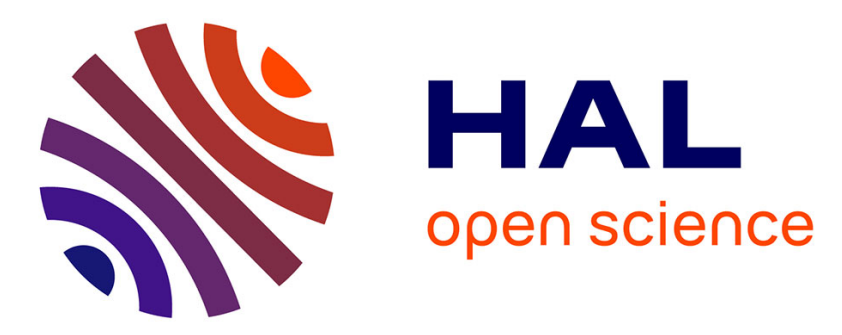

\title{
Analysis of variational formulations and low-regularity solutions for time-harmonic electromagnetic problems in complex anisotropic media
}

\author{
Damien Chicaud, Patrick Ciarlet, Axel Modave
}

\section{- To cite this version:}

Damien Chicaud, Patrick Ciarlet, Axel Modave. Analysis of variational formulations and lowregularity solutions for time-harmonic electromagnetic problems in complex anisotropic media. SIAM Journal on Mathematical Analysis, 2021, 53 (3), pp.2691-2717. 10.1137/20M1344111. hal$02651682 \mathrm{v} 2$

\section{HAL Id: hal-02651682 \\ https://hal.science/hal-02651682v2}

Submitted on 29 Jun 2021 (v2), last revised 17 Dec 2021 (v3)

HAL is a multi-disciplinary open access archive for the deposit and dissemination of scientific research documents, whether they are published or not. The documents may come from teaching and research institutions in France or abroad, or from public or private research centers.
L'archive ouverte pluridisciplinaire HAL, est destinée au dépôt et à la diffusion de documents scientifiques de niveau recherche, publiés ou non, émanant des établissements d'enseignement et de recherche français ou étrangers, des laboratoires publics ou privés. 


\title{
Analysis of variational formulations and low-regularity solutions for time-harmonic electromagnetic problems in complex anisotropic media*
}

\author{
Damien Chicaud ${ }^{1}$, Patrick Ciarlet, Jr. ${ }^{1}$, and Axel Modave ${ }^{1}$ \\ ${ }^{1}$ POEMS, CNRS, INRIA, ENSTA Paris, Institut Polytechnique de Paris, 828 Bd des Maréchaux, 91120 \\ Palaiseau, France. E-mail addresses: damien.chicaud@ensta-paris.fr, patrick.ciarlet@ensta-paris.fr, \\ axel.modave@ensta-paris.fr
}

\begin{abstract}
We consider the time-harmonic Maxwell's equations with physical parameters, namely the electric permittivity and the magnetic permeability, that are complex, possibly nonHermitian, tensor fields. Both tensor fields verify a general ellipticity condition. In this work, the well-posedness of formulations for the Dirichlet and Neumann problems (i.e. with a boundary condition on the electric field or its curl, respectively) is proven using well-suited function spaces and Helmholtz decompositions. For both problems, the a priori regularity of the solution and the solution's curl is analysed. The regularity results are obtained by splitting the fields and using shift theorems for second-order divergence elliptic operators. Finally, the discretization of the formulations with a $\boldsymbol{H}(\mathbf{c u r l})$-conforming approximation based on edge finite elements is considered. An a priori error estimate is derived and verified thanks to numerical results with an elementary benchmark.
\end{abstract}

Maxwell's Equations; Wave Propagation; Anisotropic media; Well-posedness; Regularity Analysis; Edge Finite Elements

AMS Subject Classification: 35J57, 65N30, $78 M 10$

\section{Introduction}

The study of linear differential models for time-harmonic electromagnetic wave propagation is a popular field. The mathematical analysis of these models has been performed for isotropic materials and certain classes of anisotropic materials. In particular, the well-posedness of boundary value problems and the regularity of their solutions have been studied with strong assumptions on the electric permittivity tensor field $\underline{\varepsilon}$ and the magnetic permeability tensor field $\boldsymbol{\mu}$ (e.g. real-valued isotropic tensors [13] and symmetric positive definite tensors [21, 8, 10]). The mathematical analysis of electromagnetic fields in anisotropic media has received some attention in recent years. The well-posedness of variational formulations with non-Hermitian material tensors has been studied e.g. in [3] for complex symmetric tensors, in [6] for particular anisotropic media coming from plasma theory, and in [27] for material tensors with an elliptic real part.

${ }^{*}$ Published in SIAM Journal on Mathematical Analysis: https://doi.org/10.1137/20M1344111 
Regularity results have been obtained for a general class of non-Hermitian material tensors with low-regularity assumptions in Ref. $[2,1,26]$. In these works, the time-harmonic Maxwell's equations are supplemented with a Dirichlet boundary condition.

The main aim of this work is to provide a detailed analysis of time-harmonic electromagnetic boundary value problems with low-regularity solutions for a general class of material tensors: The electric permittivity and the magnetic permeability are assumed to be complex tensor fields, possibly non-Hermitian, that fulfill a general ellipticity condition. We consider variational formulations with the electric field as unknown, with a boundary condition on the field itself or on its curl, which correspond to the so-called Dirichlet and Neumann cases, respectively. The well-posedness and the a priori regularity of the solution and the solution's curl are studied for both cases. In our framework, the regularity estimates depend crucially on the regularity of the data.

The numerical solution of electromagnetic problems is naturally performed with edge finite element methods, which lead to $\boldsymbol{H}$ (curl)-conforming approximations. While the comprehensive numerical analysis of the approximate problems is out of the scope of this paper, a preliminary analysis is proposed. In the coercive case, an a priori error estimate is derived by leveraging the regularity results obtained for the exact problems. A numerical illustration with a simple manufactured benchmark is presented as well.

This paper is organized as follows. In section 2, we extend classical results of functional analysis required for the analyses. In sections 3 and 4, we propose the analysis of the problems supplemented by Dirichlet or Neumann boundary conditions, respectively. In section 5, we address the discretization with edge finite elements. A conclusion and extensions are proposed in section 6 .

\section{Notation and hypotheses}

Vector fields are written in boldface characters, and tensor fields are written in underlined bold characters. Given a non-empty open set $\mathcal{O}$ of $\mathbb{R}^{3}$, we use the notation $(\cdot \mid \cdot)_{0, \mathcal{O}}\left(\right.$ resp. $\left.\|\cdot\|_{0, \mathcal{O}}\right)$ for the $L^{2}(\mathcal{O})$ and the $\boldsymbol{L}^{2}(\mathcal{O}):=\left(L^{2}(\mathcal{O})\right)^{3}$ scalar products (resp. norms). More generally, $(\cdot \mid \cdot)_{\mathbf{s}, \mathcal{O}}$ and $\|\cdot\|_{\mathbf{s}, \mathcal{O}}\left(\right.$ resp. $\left.|\cdot|_{\mathbf{s}, \mathcal{O}}\right)$ denote the scalar product and the norm (resp. seminorm) of the Sobolev spaces $H^{\mathbf{s}}(\mathcal{O})$ and $\boldsymbol{H}^{\mathbf{s}}(\mathcal{O}):=\left(H^{\mathbf{s}}(\mathcal{O})\right)^{3}$ for $\mathbf{s} \in \mathbb{R}$ (resp. for $\mathbf{s}>0$ ). We use the notation $\langle u, v\rangle_{H^{\mathrm{s}}(\mathcal{O})}$ for the duality product of $u \in\left(H^{\mathbf{s}}(\mathcal{O})\right)^{\prime}$ and $v \in H^{\mathbf{s}}(\mathcal{O})$. The space $H_{\mathrm{zmv}}^{\mathrm{s}}(\mathcal{O})$ is the subspace of $H^{\mathrm{s}}(\mathcal{O})$ with zero-mean-value fields. It is assumed that the reader is familiar with the function spaces related to Maxwell's equations, such as $\boldsymbol{H}(\mathbf{c u r l} ; \mathcal{O})$, $\boldsymbol{H}_{0}(\boldsymbol{c u r l} ; \mathcal{O}), \boldsymbol{H}(\operatorname{div} ; \mathcal{O})$ and $\boldsymbol{H}_{0}(\operatorname{div} ; \mathcal{O})$. A priori, $\boldsymbol{H}(\mathbf{c u r l} ; \mathcal{O})$ is endowed with the "natural" norm $\boldsymbol{v} \mapsto\|\boldsymbol{v}\|_{\boldsymbol{H}(\mathbf{c u r l} ; \mathcal{O})}:=\left(\|\boldsymbol{v}\|_{0, \mathcal{O}}^{2}+\|\operatorname{curl} \boldsymbol{v}\|_{0, \mathcal{O}}^{2}\right)^{1 / 2}$. We refer to the monographs of Monk [24], Kirsch and Hettlich [23], and Assous et al. [5] for further details.

The symbol $C$ is used to denote a generic positive constant which is independent of the mesh and the fields of interest; $C$ may depend on the geometry, or on the coefficients defining the model. We use the notation $A \lesssim B$ for the inequality $A \leq C B$, where $A$ and $B$ are two scalar fields, and $C$ is a generic constant.

In this work, $\Omega$ is assumed to be an open, connected, bounded domain of $\mathbb{R}^{3}$, with a Lipschitzcontinuous boundary $\partial \Omega$. In addition, the boundary is assumed to be of class $\mathcal{C}^{2}$ in the sections dedicated to the regularity analysis (in subsections 3.2, 3.3, 4.2 and 4.3). The unit outward normal vector field to $\partial \Omega$ is denoted $\boldsymbol{n}$. We recall the classical integration by parts formula (see Eq. (35) in Ref. [9]):

$$
(\boldsymbol{u} \mid \operatorname{curl} \boldsymbol{v})_{0, \Omega}-(\operatorname{curl} \boldsymbol{u} \mid \boldsymbol{v})_{0, \Omega}={ }_{\gamma}\left\langle\gamma^{T}(\boldsymbol{u}), \pi^{T}(\boldsymbol{v})\right\rangle_{\pi}, \forall \boldsymbol{u}, \boldsymbol{v} \in \boldsymbol{H}(\operatorname{curl} ; \Omega),
$$


where $\gamma^{T}: \boldsymbol{u} \mapsto(\boldsymbol{u} \times \boldsymbol{n})_{\mid \partial \Omega}$ denotes the tangential trace operator, $\pi^{T}: \boldsymbol{v} \mapsto \boldsymbol{n} \times(\boldsymbol{v} \times \boldsymbol{n})_{\mid \partial \Omega}$ denotes the tangential components trace operator, and $\gamma_{\gamma}\left\langle\gamma^{T}(\boldsymbol{u}), \pi^{T}(\boldsymbol{v})\right\rangle_{\pi}$ expresses duality between the ad hoc trace spaces. We denote $\left\{\Gamma_{k}\right\}_{1 \leq k \leq K}$ the maximal connected components of $\partial \Omega$. Topologically speaking, the domain $\Omega$ can be trivial or non-trivial [17] under assumptions given in Appendix A. Whenever this knowledge is needed, we use the notation (Top) ${ }_{I}$, with $I=0$ for a topologically trivial domain and $I>0$ for a topologically non-trivial domain.

\section{Model and extended functional framework}

We consider the time-harmonic Maxwell's equation, posed in $\Omega$ :

$$
\operatorname{curl}\left(\underline{\boldsymbol{\mu}}^{-1} \operatorname{curl} \boldsymbol{E}\right)-\omega^{2} \underline{\varepsilon} \boldsymbol{E}=\boldsymbol{f}
$$

where our unknown is the electric field $\boldsymbol{E}$, which we assume a priori to belong to $\boldsymbol{H}(\mathbf{c u r l} ; \Omega)$; $\underline{\varepsilon}$ and $\boldsymbol{\mu}$ are respectively the electric permittivity tensor and the magnetic permeability tensor; $\omega>0$ is the angular frequency, and $\boldsymbol{f}$ is a volume data. The problem shall be supplemented by appropriate boundary conditions on $\partial \Omega$.

In this work, the material tensors $\underline{\varepsilon}$ and $\underline{\mu}$ are assumed to satisfy an ellipticity condition. This condition is defined in the following subsection, and useful technical properties are given. The remainder of the section is dedicated to the introduction of the framework that derives from the ellipticity condition, and the extension of classical results (i.e. for elliptic, Hermitian tensor fields) given in Ref. [5]: Helmholtz decompositions, Weber inequalities, and compact embedding results.

\subsection{Ellipticity condition}

Definition 2.1. We say that a complex-valued tensor field $\underline{\boldsymbol{\xi}} \in \underline{\mathbf{L}}^{\infty}(\Omega)$ is elliptic if and only if

$$
\exists \theta_{\xi} \in \mathbb{R}, \exists \xi_{-}>0, \forall \boldsymbol{z} \in \mathbb{C}^{3}, \xi_{-}|\boldsymbol{z}|^{2} \leq \Re\left[e^{i \theta_{\xi}} \cdot \boldsymbol{z}^{*} \underline{\boldsymbol{\xi}}\right] \quad \text { almost everywhere in } \Omega .
$$

In addition, we will use the notation $\xi_{+}:=\|\underline{\boldsymbol{\xi}}\|_{\mathbf{L}^{\infty}(\Omega)}=\sup _{i, j}\left\|\xi_{i j}\right\|_{L^{\infty}(\Omega)}$, where $\left(\xi_{i j}\right)$ are the components of $\underline{\boldsymbol{\xi}}$.

This condition means that, almost everywhere in $\Omega$, the eigenvalues of $\underline{\boldsymbol{\xi}}(\boldsymbol{x})$ are contained in a fixed open half-plane of $\mathbb{C}^{3}$; in other words, there exists a "coercivity direction" for $\underline{\xi}$ in the complex plane. This condition is slightly more general than the one proposed e.g. by [27], varying only by a phase factor. This allows us to cover a more general class of material properties, such as material tensors coming from plasma theory (e.g. in [6]) which do not satisfy the standard ellipticity condition used in [27], but which are elliptic for well-suited phase factors according to Definition 2.1. Because $\underline{\boldsymbol{\xi}}$ can be non-Hermitian, the mapping $(\boldsymbol{v}, \boldsymbol{w}) \mapsto(\underline{\boldsymbol{\xi}} \boldsymbol{v} \mid \boldsymbol{w})_{0, \Omega}$ is, in general, not a scalar product in $\boldsymbol{L}^{2}(\Omega)$; orthogonality properties are lateralized, in the sense that $(\underline{\boldsymbol{\xi}} \boldsymbol{v} \mid \boldsymbol{w})_{0, \Omega}=0$ is not equivalent to $(\underline{\boldsymbol{\xi}} \boldsymbol{w} \mid \boldsymbol{v})_{0, \Omega}=0$.

Assumption. In the remainder of Section 2, the tensor $\underline{\boldsymbol{\xi}}$ belongs to $\underline{\mathbf{L}}^{\infty}(\Omega)$ and satisfies the ellipticity condition.

Proposition 2.1. One has $\underline{\boldsymbol{\xi}}^{-1} \in \underline{\mathbf{L}}^{\infty}(\Omega)$ with $\left(\xi^{-1}\right)_{+} \leq\left(\xi_{-}\right)^{-1}$. Moreover, $\underline{\boldsymbol{\xi}}^{-1}$ satisfies the ellipticity condition with $\theta_{\xi^{-1}}=-\theta_{\xi}$, and $\left(\xi^{-1}\right)_{-}:=\xi_{-}\left(\xi_{+}\right)^{-2}$. 
Proposition 2.2. For any $\boldsymbol{v} \in \boldsymbol{L}^{2}(\Omega)$, one has the following inequalities:

$$
\xi_{-}\|\boldsymbol{v}\|_{0, \Omega}^{2} \leq \Re\left[e^{i \theta_{\xi}}(\underline{\boldsymbol{\xi}} \boldsymbol{v} \mid \boldsymbol{v})_{0, \Omega}\right] \leq\left|(\underline{\boldsymbol{\xi}} \boldsymbol{v} \mid \boldsymbol{v})_{0, \Omega}\right| \leq \xi_{+}\|\boldsymbol{v}\|_{0, \Omega}^{2}
$$

Then, the ellipticity condition implies that the inverse of the second-order divergence elliptic operators, with Dirichlet or Neumann boundary condition, is well-defined. Equivalently, this implies the well-posedness of the problems below, called either the Dirichlet problem or the Neumann problem from now on.

Theorem 2.1. Under assumption (2), the Dirichlet problem

$$
\left\{\begin{array}{l}
\text { Find } p \in H_{0}^{1}(\Omega) \text { such that } \\
(\underline{\boldsymbol{\xi}} \nabla p \mid \nabla q)_{0, \Omega}=\ell(q), \forall q \in H_{0}^{1}(\Omega),
\end{array}\right.
$$

is well-posed for all $\ell$ in $H^{-1}(\Omega)=\left(H_{0}^{1}(\Omega)\right)^{\prime}$, that is

$$
\exists C>0, \forall \ell \in\left(H_{0}^{1}(\Omega)\right)^{\prime}, \exists \text { ! p solution to (4), with }\|p\|_{1, \Omega} \leq C\|\ell\|_{\left(H_{0}^{1}(\Omega)\right)^{\prime}} .
$$

Similarly, under assumption (2), the Neumann problem

$$
\left\{\begin{array}{l}
\text { Find } p \in H_{\mathrm{zmv}}^{1}(\Omega) \text { such that } \\
(\underline{\boldsymbol{\xi}} \nabla p \mid \nabla q)_{0, \Omega}=\ell(q), \forall q \in H_{\mathrm{zmv}}^{1}(\Omega),
\end{array}\right.
$$

is well-posed for all $\ell$ in $\left(H_{\mathrm{zmv}}^{1}(\Omega)\right)^{\prime}$, that is

$$
\exists C>0, \forall \ell \in\left(H_{\mathrm{zmv}}^{1}(\Omega)\right)^{\prime}, \exists ! p \text { solution to }(5) \text {, with }\|p\|_{1, \Omega} \leq C\|\ell\|_{\left.\left(H_{\mathrm{zmv}}^{1}(\Omega)\right)\right)^{\prime}} .
$$

Proof. One uses Lax-Milgram's theorem. All assumptions are fulfilled, and in particular coercivity of the sesquilinear form follows from relations (3) and Poincaré, resp. Poincaré-Wirtinger, inequalities.

\subsection{Helmholtz decompositions}

Definition 2.2. We introduce the function spaces:

$$
\begin{aligned}
& \boldsymbol{H}(\operatorname{div} \underline{\boldsymbol{\xi}} ; \Omega):=\left\{\boldsymbol{v} \in \boldsymbol{L}^{2}(\Omega), \underline{\boldsymbol{\xi} \boldsymbol{v}} \in \boldsymbol{H}(\operatorname{div} ; \Omega)\right\}, \\
& \boldsymbol{H}_{0}(\operatorname{div} \underline{\boldsymbol{\xi}} ; \Omega):=\left\{\boldsymbol{v} \in \boldsymbol{L}^{2}(\Omega), \underline{\boldsymbol{\xi}} \boldsymbol{v} \in \boldsymbol{H}_{0}(\operatorname{div} ; \Omega)\right\}, \\
& \boldsymbol{H}(\operatorname{div} \underline{\boldsymbol{\xi}} 0 ; \Omega):=\{\boldsymbol{v} \in \boldsymbol{H}(\operatorname{div} \underline{\boldsymbol{\xi}} ; \Omega), \operatorname{div} \underline{\boldsymbol{\xi}} \boldsymbol{v}=0\}, \\
& \boldsymbol{H}_{0}(\operatorname{div} \underline{\boldsymbol{\xi}} 0 ; \Omega):=\boldsymbol{H}_{0}(\operatorname{div} \underline{\boldsymbol{\xi}} ; \Omega) \cap \boldsymbol{H}(\operatorname{div} \underline{\boldsymbol{\xi}} 0 ; \Omega) \text {, }
\end{aligned}
$$

as well as

$$
\begin{aligned}
\boldsymbol{X}_{N}(\underline{\boldsymbol{\xi}} ; \Omega) & :=\boldsymbol{H}_{0}(\operatorname{curl} ; \Omega) \cap \boldsymbol{H}(\operatorname{div} \underline{\boldsymbol{\xi}} ; \Omega), \\
\boldsymbol{X}_{T}(\underline{\boldsymbol{\xi}} ; \Omega) & :=\boldsymbol{H}(\mathbf{\operatorname { c u r l }} ; \Omega) \cap \boldsymbol{H}_{0}(\operatorname{div} \underline{\boldsymbol{\xi}} ; \Omega), \\
\boldsymbol{K}_{N}(\underline{\boldsymbol{\xi}} ; \Omega) & :=\boldsymbol{H}_{0}(\operatorname{curl} ; \Omega) \cap \boldsymbol{H}(\operatorname{div} \underline{\boldsymbol{\xi}} 0 ; \Omega), \\
\boldsymbol{K}_{T}(\underline{\boldsymbol{\xi}} ; \Omega) & :=\boldsymbol{H}(\boldsymbol{\operatorname { c u r l }} ; \Omega) \cap \boldsymbol{H}_{0}(\operatorname{div} \underline{\boldsymbol{\xi}} 0 ; \Omega) .
\end{aligned}
$$

The function spaces $\boldsymbol{X}_{N}(\underline{\boldsymbol{\xi}} ; \Omega), \boldsymbol{X}_{T}(\underline{\boldsymbol{\xi}} ; \Omega), \boldsymbol{K}_{N}(\underline{\boldsymbol{\xi}} ; \Omega)$ and $\boldsymbol{K}_{T}(\underline{\boldsymbol{\xi}} ; \Omega)$ are endowed with the graph norm $\boldsymbol{v} \mapsto\left(\|\boldsymbol{v}\|_{\boldsymbol{H}(\mathbf{c u r l} ; \Omega)}^{2}+\|\underline{\boldsymbol{\xi}}\|_{\boldsymbol{H}(\mathrm{div} ; \Omega)}^{2}\right)^{1 / 2}$. When $\underline{\boldsymbol{\xi}}$ is equal to the identity tensor $\mathbb{I}_{3}$, we choose the simpler notation $\boldsymbol{X}_{N}(\Omega)$ instead of $\boldsymbol{X}_{N}\left(\mathbb{I}_{3} ; \Omega\right)$, etc. 
As a first noticeable consequence of (2), one can prove the Helmholtz decompositions below.

Theorem 2.2. Under assumption (2), one has the first-kind Helmholtz decompositions:

$$
\begin{aligned}
& \boldsymbol{L}^{2}(\Omega)=\nabla H_{0}^{1}(\Omega) \oplus \boldsymbol{H}(\operatorname{div} \underline{\boldsymbol{\xi}} 0 ; \Omega) ; \\
& \boldsymbol{H}_{0}(\mathbf{\operatorname { c u r l }} ; \Omega)=\nabla H_{0}^{1}(\Omega) \oplus \boldsymbol{K}_{N}(\underline{\boldsymbol{\xi}} ; \Omega) .
\end{aligned}
$$

In addition, these sums are continuous.

Proof. Let $\boldsymbol{v} \in \boldsymbol{L}^{2}(\Omega)$. The Dirichlet problem

$$
\left\{\begin{array}{l}
\text { Find } p \in H_{0}^{1}(\Omega) \text { such that } \\
(\underline{\boldsymbol{\xi}} \nabla p \mid \nabla q)_{0, \Omega}=(\underline{\boldsymbol{\xi}} \boldsymbol{v} \mid \nabla q)_{0, \Omega}, \forall q \in H_{0}^{1}(\Omega),
\end{array}\right.
$$

is well-posed by theorem 2.1. Let $\boldsymbol{v}_{T}=\boldsymbol{v}-\nabla p \in \boldsymbol{L}^{2}(\Omega)$. Then $\operatorname{div} \underline{\boldsymbol{\xi}} \boldsymbol{v}_{T}=\operatorname{div} \underline{\boldsymbol{\xi}} \boldsymbol{v}-\operatorname{div} \underline{\boldsymbol{\xi}} \nabla p=0$, i.e. $\boldsymbol{v}_{T} \in \boldsymbol{H}(\operatorname{div} \boldsymbol{\xi} 0 ; \Omega)$.

Additionally, the sum is direct: indeed, let $\boldsymbol{v} \in \nabla H_{0}^{1}(\Omega) \cap \boldsymbol{H}(\operatorname{div} \boldsymbol{\xi} 0 ; \Omega)$, then $\boldsymbol{v}=\nabla p$ for some $p \in H_{0}^{1}(\Omega)$, and $\operatorname{div} \underline{\boldsymbol{\xi}} \nabla p=0$. But the Dirichlet problem is well-posed, so $p=0$.

Finally, we note that, thanks to theorem $2.1, \nabla p$ depends continuously on $\boldsymbol{v}:\|\nabla p\|_{0, \Omega} \lesssim\|\boldsymbol{v}\|_{0, \Omega}$. It is also the case for $\boldsymbol{v}_{T}$ by the triangle inequality: $\left\|\boldsymbol{v}_{T}\right\|_{0, \Omega} \leq\|\nabla p\|_{0, \Omega}+\|\boldsymbol{v}\|_{0, \Omega} \lesssim\|\boldsymbol{v}\|_{0, \Omega}$.

The second proof is similar, with bounds in $\left.\|\cdot\|_{\boldsymbol{H}(\mathbf{c u r l} ; \Omega)}\right)^{- \text {norm. }}$

Theorem 2.3. Under assumption (2), one has the second-kind Helmholtz decompositions:

$$
\begin{aligned}
& \boldsymbol{L}^{2}(\Omega)=\nabla H_{\mathrm{zmv}}^{1}(\Omega) \oplus \boldsymbol{H}_{0}(\operatorname{div} \underline{\boldsymbol{\xi}} 0 ; \Omega) ; \\
& \boldsymbol{H}(\mathbf{c u r l} ; \Omega)=\nabla H_{\mathrm{zmv}}^{1}(\Omega) \oplus \boldsymbol{K}_{T}(\underline{\boldsymbol{\xi}} ; \Omega) .
\end{aligned}
$$

In addition, these sums are continuous.

Proof. Let $\boldsymbol{v} \in \boldsymbol{L}^{2}(\Omega)$. The Neumann problem

$$
\left\{\begin{array}{l}
\text { Find } p \in H_{\mathrm{zmv}}^{1}(\Omega) \text { such that } \\
(\underline{\boldsymbol{\xi}} \nabla p \mid \nabla q)_{0, \Omega}=(\underline{\boldsymbol{\xi}} \boldsymbol{v} \mid \nabla q)_{0, \Omega}, \forall q \in H_{\mathrm{zmv}}^{1}(\Omega),
\end{array}\right.
$$

is well-posed by theorem 2.1. We pose $\boldsymbol{v}_{T}=\boldsymbol{v}-\nabla p \in \boldsymbol{L}^{2}(\Omega)$. Noting that the formulation is still valid for all $q \in H^{1}(\Omega)$, and taking $q \in H_{0}^{1}(\Omega)$, there holds $\left\langle\operatorname{div} \underline{\boldsymbol{\xi}} \boldsymbol{v}_{T}, q\right\rangle_{H_{0}^{1}(\Omega)}=-\left(\underline{\boldsymbol{\xi}} \boldsymbol{v}_{T} \mid \nabla q\right)_{0, \Omega}=$ 0 . Hence $\operatorname{div} \underline{\boldsymbol{\xi}} \boldsymbol{v}_{T}=0$ and $\boldsymbol{v}_{T} \in \boldsymbol{H}(\operatorname{div} \underline{\boldsymbol{\xi}} 0 ; \Omega)$. Moreover, $\forall q \in H^{1}(\Omega),\left\langle\underline{\boldsymbol{\xi}} \boldsymbol{v}_{T} \cdot \boldsymbol{n}, q\right\rangle_{H^{1 / 2}(\partial \Omega)}=$ $\left(\underline{\boldsymbol{\xi}} \boldsymbol{v}_{T} \mid \nabla q\right)_{0, \Omega}+\left(\operatorname{div} \underline{\boldsymbol{\xi}} \boldsymbol{v}_{T} \mid q\right)_{0, \Omega}=0$. Hence $\boldsymbol{v}_{T} \in \boldsymbol{H}_{0}(\operatorname{div} \underline{\boldsymbol{\xi}} 0 ; \Omega)$.

Additionally, the sum is direct: indeed, let $\boldsymbol{v} \in \nabla H_{\mathrm{zmv}}^{1}(\Omega) \cap \boldsymbol{H}_{0}(\operatorname{div} \boldsymbol{\xi} 0 ; \Omega)$, then $\boldsymbol{v}=\nabla p$ for some $p \in H_{\mathrm{zmv}}^{1}(\Omega)$, and fulfills $\operatorname{div} \underline{\boldsymbol{\xi}} \nabla p=0$ and $\underline{\boldsymbol{\xi}} \nabla p \cdot \boldsymbol{n}_{\mid \partial \Omega}=0$. As the Neumann problem is well-posed, $p=0$. The fact that the sum is continuous is derived as previously.

The second proof is similar.

Remark 2.1. We recall that the notion of orthogonality no longer applies, as the mapping $(\underline{\boldsymbol{\xi}} \cdot \mid \cdot)_{0, \Omega}$ is not automatically a scalar product. For instance, for $\boldsymbol{v} \in \boldsymbol{H}(\operatorname{div} \underline{\boldsymbol{\xi}} 0 ; \Omega), q \in H_{0}^{1}(\Omega)$, it always holds that $(\underline{\boldsymbol{\xi}} \boldsymbol{v} \mid \nabla q)_{0, \Omega}=0$ by integration by parts. On the other hand, $(\underline{\boldsymbol{\xi}} \nabla q \mid \boldsymbol{v})_{0, \Omega}$ may not vanish. 


\subsection{The function space $\boldsymbol{X}_{N}(\underline{\xi} ; \Omega)$}

Let us begin with an extension of the first Weber inequality, cf. Theorem 6.1.6 in Ref. [5].

Theorem 2.4. Under assumption (2), one has the Weber inequality

$$
\begin{aligned}
& \exists C_{W}>0, \forall \boldsymbol{y} \in \boldsymbol{X}_{N}(\underline{\boldsymbol{\xi}} ; \Omega) \\
& \|\boldsymbol{y}\|_{0, \Omega} \leq C_{W}\left(\|\operatorname{curl} \boldsymbol{y}\|_{0, \Omega}+\|\operatorname{div} \underline{\boldsymbol{\xi}}\|_{0, \Omega}+\sum_{1 \leq k \leq K}\left|\langle\underline{\boldsymbol{\xi}} \cdot \boldsymbol{n}, 1\rangle_{H^{1 / 2}\left(\Gamma_{k}\right)}\right|\right),
\end{aligned}
$$

Proof. We proceed by contradiction. Let us assume there exists $\left(\boldsymbol{y}_{m}\right)$ a sequence of $\boldsymbol{X}_{N}(\underline{\boldsymbol{\xi}} ; \Omega)$ such that, $\forall m,\left\|\boldsymbol{y}_{m}\right\|_{0, \Omega}=1$ and

$$
\left\|\operatorname{curl} \boldsymbol{y}_{m}\right\|_{0, \Omega}+\left\|\operatorname{div} \underline{\boldsymbol{\xi}} \boldsymbol{y}_{m}\right\|_{0, \Omega}+\sum_{1 \leq k \leq K}\left|\left\langle\underline{\boldsymbol{\xi}} \boldsymbol{y}_{m} \cdot \boldsymbol{n}, 1\right\rangle_{H^{1 / 2}\left(\Gamma_{k}\right)}\right| \leq \frac{1}{m+1} .
$$

Step 1. Consider the solution to the Dirichlet problem

$$
\left\{\begin{array}{l}
\text { Find } q_{m}^{0} \in H_{0}^{1}(\Omega) \text { such that } \\
\left(\underline{\boldsymbol{\xi}} \nabla q_{m}^{0} \mid \nabla q\right)_{0, \Omega}=\left(\underline{\boldsymbol{\xi}} \boldsymbol{y}_{m} \mid \nabla q\right)_{0, \Omega}, \forall q \in H_{0}^{1}(\Omega),
\end{array}\right.
$$

By theorem 2.1, this problem is well-posed. Moreover, taking $q=q_{m}^{0}$, one gets

$$
\left|\left(\underline{\boldsymbol{\xi}} \nabla q_{m}^{0} \mid \nabla q_{m}^{0}\right)_{0, \Omega}\right|=\left|\left(-\operatorname{div} \underline{\boldsymbol{\xi}} \boldsymbol{y}_{m} \mid q_{m}^{0}\right)_{0, \Omega}\right| \leq\left\|\operatorname{div} \underline{\boldsymbol{\xi}} \boldsymbol{y}_{m}\right\|_{0, \Omega}\left\|q_{m}^{0}\right\|_{0, \Omega} .
$$

Using the relation (3) on the left-hand side and the Poincaré inequality on the right-hand side, one gets $\left\|\nabla q_{m}^{0}\right\|_{0, \Omega}^{2} \lesssim\left\|\operatorname{div} \underline{\boldsymbol{\xi}} \boldsymbol{y}_{m}\right\|_{0, \Omega}\left\|\nabla q_{m}^{0}\right\|_{0, \Omega}$, so

$$
\left\|\nabla q_{m}^{0}\right\|_{0, \Omega} \lesssim\left\|\operatorname{div} \underline{\boldsymbol{\xi}} \boldsymbol{y}_{m}\right\|_{0, \Omega}
$$

One gets that $\left\|\nabla q_{m}^{0}\right\|_{0, \Omega} \longrightarrow 0$.

Step 2. Let $\boldsymbol{x}_{m}:=\boldsymbol{y}_{m}-\nabla q_{m}^{0} \in \boldsymbol{K}_{N}(\underline{\xi} ; \Omega)$ (this is the Helmholtz decomposition (7) of $\boldsymbol{y}_{m}$ ). Consider the finite-dimensional space $\bar{Q}_{N}(\underline{\xi} ; \Omega):=\left\{q \in H^{1}(\Omega) \mid \operatorname{div} \underline{\boldsymbol{\xi}} \nabla q=0\right.$ in $\Omega, q_{\mid \Gamma_{0}}=$ $0, q_{\mid \Gamma_{k}}=$ cst $\left._{k}, 1 \leq k \leq K\right\}$, where cst $_{k}$ is a constant field on $\Gamma_{k}$, and the solution to the problem

$$
\left\{\begin{array}{l}
\text { Find } q_{m}^{\Gamma} \in Q_{N}(\underline{\boldsymbol{\xi}} ; \Omega) \text { such that } \\
\left(\underline{\boldsymbol{\xi}} \nabla q_{m}^{\Gamma} \mid \nabla q\right)_{0, \Omega} \stackrel{=}{=}\left(\underline{\boldsymbol{\xi}} \boldsymbol{x}_{m} \mid \nabla q\right)_{0, \Omega}, \forall q \in Q_{N}(\underline{\boldsymbol{\xi}} ; \Omega) .
\end{array}\right.
$$

This problem is also well-posed, following the proof of theorem 2.1 with the Poincaré inequality in $\left\{q \in H^{1}(\Omega), q_{\mid \Gamma_{0}}=0\right\}$. Taking $q=q_{m}^{\Gamma}$ and integrating by parts, one has

$$
\begin{aligned}
\left|\left(\underline{\boldsymbol{\xi}} \nabla q_{m}^{\Gamma} \mid \nabla q_{m}^{\Gamma}\right)_{0, \Omega}\right| & =\left|\left\langle\underline{\boldsymbol{\xi}} \boldsymbol{x}_{m} \cdot \boldsymbol{n}, q_{m}^{\Gamma}\right\rangle_{H^{1 / 2}(\partial \Omega)}\right| \\
& =\left|\sum_{1 \leq k \leq K}\left\langle\underline{\boldsymbol{\xi}} \boldsymbol{x}_{m} \cdot \boldsymbol{n}, q_{m}^{\Gamma}\right\rangle_{H^{1 / 2}\left(\Gamma_{k}\right)}\right| \\
& =\left|\sum_{1 \leq k \leq K} \overline{q_{m \mid \Gamma_{k}}^{\Gamma}}\left\langle\underline{\boldsymbol{\xi}} \boldsymbol{x}_{m} \cdot \boldsymbol{n}, 1\right\rangle_{H^{1 / 2}\left(\Gamma_{k}\right)}\right| .
\end{aligned}
$$

As $Q_{N}(\underline{\boldsymbol{\xi}} ; \Omega)$ is a finite-dimensional vector space, all the norms are equivalent, and among them, $\|\nabla \cdot\|_{0, \Omega}$ and $\max _{k}|\cdot| \Gamma_{k} \mid$. Then, using additionally the relation (3), there holds

$$
\left\|q_{m}^{\Gamma}\right\|_{Q_{N}(\underline{\boldsymbol{\xi}})}^{2} \lesssim\left(\left\|q_{m}^{\Gamma}\right\|_{Q_{N}(\underline{\boldsymbol{\xi}})} \sum_{1 \leq k \leq K}\left|\left\langle\underline{\boldsymbol{\xi}} \boldsymbol{x}_{m} \cdot \boldsymbol{n}, 1\right\rangle_{H^{1 / 2}\left(\Gamma_{k}\right)}\right|\right) .
$$


In addition, $\left\langle\underline{\boldsymbol{\xi}} \boldsymbol{x}_{m} \cdot \boldsymbol{n}, 1\right\rangle_{H^{1 / 2}\left(\Gamma_{k}\right)}=\left\langle\underline{\boldsymbol{\xi}} \boldsymbol{y}_{m} \cdot \boldsymbol{n}, 1\right\rangle_{H^{1 / 2}\left(\Gamma_{k}\right)}-\left\langle\underline{\boldsymbol{\xi}} \nabla q_{m}^{0} \cdot \boldsymbol{n}, 1\right\rangle_{H^{1 / 2}\left(\Gamma_{k}\right)}$, and, using the continuity of the normal trace and recalling that $\operatorname{div} \underline{\boldsymbol{\xi}} \nabla q_{m}^{0}=\operatorname{div} \underline{\boldsymbol{\xi}} \boldsymbol{y}_{m}$ as well as relation (12), one obtains

$$
\left|\left\langle\underline{\boldsymbol{\xi}} \nabla q_{m}^{0} \cdot \boldsymbol{n}, 1\right\rangle_{H^{1 / 2}\left(\Gamma_{k}\right)}\right| \lesssim\left\|\underline{\boldsymbol{\xi}} \nabla q_{m}^{0} \cdot \boldsymbol{n}\right\|_{-1 / 2, \Gamma_{k}} \lesssim\left\|\nabla q_{m}^{0}\right\|_{\boldsymbol{H}(\operatorname{div} \underline{\boldsymbol{\xi}} ; \Omega)} \lesssim\left\|\operatorname{div} \underline{\boldsymbol{\xi}} \boldsymbol{y}_{m}\right\|_{0, \Omega} .
$$

Hence

$$
\left\|q_{m}^{\Gamma}\right\|_{Q_{N}(\underline{\boldsymbol{\xi}})} \lesssim\left(\sum_{1 \leq k \leq K}\left|\left\langle\underline{\boldsymbol{\xi}} \boldsymbol{y}_{m} \cdot \boldsymbol{n}, 1\right\rangle_{H^{1 / 2}\left(\Gamma_{k}\right)}\right|+\left\|\operatorname{div} \underline{\boldsymbol{\xi}} \boldsymbol{y}_{m}\right\|_{0, \Omega}\right),
$$

and one gets that $\left\|\nabla q_{m}^{\Gamma}\right\|_{0, \Omega} \longrightarrow 0$. Observe that $\nabla q_{m}^{\Gamma} \times \boldsymbol{n}_{\mid \partial \Omega}=0$, so $\nabla q_{m}^{\Gamma} \in \boldsymbol{H}_{0}(\operatorname{curl} ; \Omega)$.

Step 3. Let $\boldsymbol{z}_{m}:=\boldsymbol{y}_{m}-\nabla q_{m}^{0}-\nabla q_{m}^{\Gamma}$. It belongs to $\boldsymbol{X}_{N}(\underline{\boldsymbol{\xi}}, \Omega)$ and, additionally, curl $\boldsymbol{z}_{m}=$ $\operatorname{curl} \boldsymbol{y}_{m}, \operatorname{div} \underline{\boldsymbol{\xi}}_{m}=0$, and $\left\langle\underline{\boldsymbol{\xi}} \boldsymbol{z}_{m} \cdot \boldsymbol{n}, 1\right\rangle_{H^{1 / 2}\left(\Gamma_{k}\right)}=0$. Indeed, introducing $q_{k}$ the unique element of $Q_{N}(\underline{\boldsymbol{\xi}} ; \Omega)$ such that $\left.q_{k}\right|_{\Gamma_{l}}=\delta_{k, l}$ for $1 \leq k \leq K$, one has by integration by parts

$$
\left\langle\underline{\boldsymbol{\xi}} \boldsymbol{z}_{m} \cdot \boldsymbol{n}, 1\right\rangle_{H^{1 / 2}\left(\Gamma_{k}\right)}=\left\langle\underline{\boldsymbol{\xi}} \boldsymbol{z}_{m} \cdot \boldsymbol{n}, q_{k}\right\rangle_{H^{1 / 2}(\partial \Omega)}=\left(\underline{\boldsymbol{\xi}} \boldsymbol{z}_{m} \mid \nabla q_{k}\right)_{0, \Omega}+\left(\operatorname{div} \underline{\boldsymbol{\xi}} \boldsymbol{z}_{m} \mid q_{k}\right)_{0, \Omega} .
$$

Moreover, $\left(\underline{\boldsymbol{\xi}} \boldsymbol{z}_{m} \mid \nabla q_{k}\right)_{0, \Omega}=\left(\underline{\boldsymbol{\xi}} \boldsymbol{x}_{m} \mid \nabla q_{k}\right)_{0, \Omega}-\left(\underline{\boldsymbol{\xi}} \nabla q_{m}^{\Gamma} \mid \nabla q_{k}\right)_{0, \Omega}=0$ by definition of $q_{m}^{\Gamma}$; and $\operatorname{div} \underline{\boldsymbol{\xi}} \boldsymbol{z}_{m}=0$. It follows that $\left\langle\underline{\boldsymbol{\xi}} \boldsymbol{z}_{m} \cdot \boldsymbol{n}, 1\right\rangle_{H^{1 / 2}\left(\Gamma_{k}\right)}=0$. With the help of the vector potential Theorem 3.4.1 [5], there exists $\boldsymbol{w}_{m} \in \boldsymbol{H}^{1}(\Omega)$ such that $\underline{\boldsymbol{\xi}} \boldsymbol{z}_{m}=\operatorname{curl} \boldsymbol{w}_{m}$, and $\left\|\boldsymbol{w}_{m}\right\|_{1, \Omega} \lesssim$ $\left\|\underline{\boldsymbol{\xi}} \boldsymbol{z}_{m}\right\|_{0, \Omega} \lesssim\left\|\boldsymbol{z}_{m}\right\|_{0, \Omega}$.

Furthermore, we have by integration by parts

$$
\left(\boldsymbol{z}_{m} \mid \underline{\boldsymbol{\xi}} \boldsymbol{z}_{m}\right)_{0, \Omega}=\left(\boldsymbol{z}_{m} \mid \operatorname{curl} \boldsymbol{w}_{m}\right)_{0, \Omega}=\left(\operatorname{curl} \boldsymbol{z}_{m} \mid \boldsymbol{w}_{m}\right)_{0, \Omega}=\left(\operatorname{curl} \boldsymbol{y}_{m} \mid \boldsymbol{w}_{m}\right)_{0, \Omega} .
$$

Using again relation (3), there holds

$$
\left\|\boldsymbol{z}_{m}\right\|_{0, \Omega}^{2} \lesssim\left\|\operatorname{curl} \boldsymbol{y}_{m}\right\|_{0, \Omega}\left\|\boldsymbol{w}_{m}\right\|_{0, \Omega} \lesssim\left\|\operatorname{curl} \boldsymbol{y}_{m}\right\|_{0, \Omega}\left\|\boldsymbol{z}_{m}\right\|_{0, \Omega}
$$

and so $\left\|\boldsymbol{z}_{m}\right\|_{0, \Omega} \lesssim\left\|\operatorname{curl} \boldsymbol{y}_{m}\right\|_{0, \Omega}$. One gets that $\left\|\boldsymbol{z}_{m}\right\|_{0, \Omega} \longrightarrow 0$.

Finally, as $\boldsymbol{y}_{m}=\boldsymbol{z}_{m}+\nabla q_{m}^{0}+\nabla q_{m}^{\Gamma}$, we have $\left\|\boldsymbol{y}_{m}\right\|_{0, \Omega} \longrightarrow 0$, which contradicts $\left\|\boldsymbol{y}_{m}\right\|_{0, \Omega}=1$ for all $m$.

One can also extend the compact embedding result of Theorem 7.5.1 in Ref. [5]. Let us note that a similar result has been proven by Alonso and Valli [3] for a different class of anisotropic materials.

Theorem 2.5. Under assumption (2), the embedding of $\boldsymbol{X}_{N}(\underline{\xi} ; \Omega)$ into $\boldsymbol{L}^{2}(\Omega)$ is compact.

Proof. Let $\left(\boldsymbol{y}_{m}\right)$ be a bounded sequence of $\boldsymbol{X}_{N}(\underline{\xi} ; \Omega)$. As in the previous proof (Steps 1., 2., 3.), we introduce $q_{m}^{0} \in H_{0}^{1}(\Omega), q_{m}^{\Gamma} \in Q_{N}(\underline{\boldsymbol{\xi}} ; \Omega)$, and $\boldsymbol{w}_{m} \in \boldsymbol{H}^{1}(\Omega)$, such that $\boldsymbol{y}_{m}=\underline{\boldsymbol{\xi}}^{-1} \operatorname{curl} \boldsymbol{w}_{m}+$ $\nabla q_{m}^{0}+\nabla q_{m}^{\Gamma}$. Additionally, there holds (see previous proof):

$$
\begin{aligned}
\left\|\nabla q_{m}^{0}\right\|_{0, \Omega} & \lesssim\left\|\operatorname{div} \underline{\boldsymbol{\xi}} \boldsymbol{y}_{m}\right\|_{0, \Omega} ; \\
\left\|\nabla q_{m}^{\Gamma}\right\|_{0, \Omega} & \lesssim\left(\left\|\operatorname{div} \underline{\boldsymbol{\xi}} \boldsymbol{y}_{m}\right\|_{0, \Omega}+\sum_{1 \leq k \leq K}\left|\left\langle\underline{\boldsymbol{\xi}} \boldsymbol{y}_{m} \cdot \boldsymbol{n}, 1\right\rangle_{H^{1 / 2}\left(\Gamma_{k}\right)}\right|\right) ; \\
\left\|\boldsymbol{w}_{m}\right\|_{1, \Omega} & \lesssim\left\|\operatorname{curl} \boldsymbol{y}_{m}\right\|_{0, \Omega} .
\end{aligned}
$$

Let us begin with $\left(q_{m}^{\Gamma}\right)$ : it is a bounded sequence of the finite-dimensional vector space $Q_{N}(\underline{\xi} ; \Omega)$, so it admits a subsequence which converges (in particular in $\|\cdot\|_{1, \Omega}$-norm). 
In addition, $\left(q_{m}^{0}\right)$ and $\left(\boldsymbol{w}_{m}\right)$ are bounded sequences of $H^{1}(\Omega)$ (resp. $\boldsymbol{H}^{1}(\Omega)$ ). Then, by Rellich's theorem, they admit susbsequences (still denoted with the same indices) which converge in $L^{2}(\Omega)$ $\left(\right.$ resp. $\left.\boldsymbol{L}^{2}(\Omega)\right)$. It remains to prove that both subsequences $\left(\nabla q_{m}^{0}\right)$ and $\left(\operatorname{curl} \boldsymbol{w}_{m}\right)$ converge in $\boldsymbol{L}^{2}(\Omega)$.

By definition of $q_{m}^{0}$, for any $q$ in $H_{0}^{1}(\Omega)$, there holds by integration by parts

$$
\left(\underline{\boldsymbol{\xi}} \nabla q_{m}^{0} \mid \nabla q\right)_{0, \Omega}=\left(\underline{\boldsymbol{\xi}} \boldsymbol{y}_{m} \mid \nabla q\right)_{0, \Omega}=-\left(\operatorname{div} \underline{\boldsymbol{\xi}} \boldsymbol{y}_{m} \mid q\right)_{0, \Omega}
$$

Using the notation $v_{m n}:=v_{m}-v_{n}$ and taking $q=q_{m n}^{0}$, one has

$$
\left|\left(\underline{\boldsymbol{\xi}} \nabla q_{m n}^{0} \mid \nabla q_{m n}^{0}\right)_{0, \Omega}\right| \leq\left\|\operatorname{div} \underline{\boldsymbol{\xi}} \boldsymbol{y}_{m n}\right\|_{0, \Omega}\left\|q_{m n}^{0}\right\|_{0, \Omega} .
$$

Then, by relation (3),

$$
\left\|\nabla q_{m n}^{0}\right\|_{0, \Omega}^{2} \lesssim 2 \sup _{m}\left(\left\|\operatorname{div} \underline{\boldsymbol{\xi}} \boldsymbol{y}_{m}\right\|_{0, \Omega}\right)\left\|q_{m n}^{0}\right\|_{0, \Omega}
$$

Thus $\left(\nabla q_{m}^{0}\right)$ is a Cauchy sequence of $\boldsymbol{L}^{2}(\Omega)$, and hence converges in this Hilbert space. We recall that $\underline{\boldsymbol{\xi}}^{-1} \operatorname{curl} \boldsymbol{w}_{m} \in \boldsymbol{X}_{N}(\underline{\boldsymbol{\xi}}, \Omega)$ (cf. Step 3. of previous proof) and $\operatorname{curl}\left(\underline{\boldsymbol{\xi}}^{-1} \operatorname{curl} \boldsymbol{w}_{m}\right)=$ $\operatorname{curl} \boldsymbol{y}_{m}$. Then, still with the same notations, and by integration by parts,

$$
\left(\underline{\boldsymbol{\xi}}^{-1} \operatorname{curl} \boldsymbol{w}_{m n} \mid \operatorname{curl} \boldsymbol{w}_{m n}\right)_{0, \Omega}=\left(\operatorname{curl}\left(\underline{\boldsymbol{\xi}}^{-1} \operatorname{curl} \boldsymbol{w}_{m n}\right) \mid \boldsymbol{w}_{m n}\right)_{0, \Omega}=\left(\operatorname{curl} \boldsymbol{y}_{m n} \mid \boldsymbol{w}_{m n}\right)_{0, \Omega} .
$$

As $\underline{\boldsymbol{\xi}}^{-1}$ also satisfies the ellipticity condition (proposition 2.1), we get

$$
\left\|\operatorname{curl} \boldsymbol{w}_{m n}\right\|_{0, \Omega}^{2} \lesssim\left\|\operatorname{curl} \boldsymbol{y}_{m n}\right\|_{0, \Omega}\left\|\boldsymbol{w}_{m n}\right\|_{0, \Omega} \leq 2 \sup _{m}\left(\left\|\operatorname{curl} \boldsymbol{y}_{m}\right\|_{0, \Omega}\right)\left\|\boldsymbol{w}_{m n}\right\|_{0, \Omega},
$$

which proves that ( $\operatorname{curl} \boldsymbol{w}_{m}$ ) is a Cauchy and hence converging sequence of $\boldsymbol{L}^{2}(\Omega)$.

As $\boldsymbol{y}_{m}=\underline{\boldsymbol{\xi}}^{-1} \operatorname{curl} \boldsymbol{w}_{m}+\nabla q_{m}^{0}+\nabla q_{m}^{\Gamma}$, we conclude that the subsequence $\left(\boldsymbol{y}_{m}\right)$ converges in $\boldsymbol{L}^{2}(\Omega)$.

\subsection{The function space $\boldsymbol{X}_{T}(\underline{\xi} ; \Omega)$}

Let us begin with an extension of the second Weber inequality, cf. Theorem 6.2.5 in Ref. [5]. Some knowledge on the topology of the domain $\Omega$ is required, see Appendix A for details and notations.

Theorem 2.6. Assume that (Top) I holds. Under assumption (2), one has the Weber inequality

$$
\begin{aligned}
& \exists C_{W}^{\prime}>0, \forall \boldsymbol{X}_{T}(\underline{\boldsymbol{\xi}} ; \Omega) \\
& \|\boldsymbol{y}\|_{0, \Omega} \leq C_{W}^{\prime}\left(\|\operatorname{curl} \boldsymbol{y}\|_{0, \Omega}+\|\operatorname{div} \underline{\boldsymbol{\xi}} \boldsymbol{y}\|_{0, \Omega}+\sum_{1 \leq i \leq I}\left|\langle\underline{\boldsymbol{\xi}} \cdot \boldsymbol{n}, 1\rangle_{H^{1 / 2}\left(\Sigma_{i}\right)}\right|\right),
\end{aligned}
$$

where $\left\{\Sigma_{i}\right\}_{1 \leq i \leq I}$ are the cuts of $\Omega$ if $I>0$ (see Appendix A).

Proof. The proof follows a similar structure as in theorem 2.4. By contradiction, we assume there exists $\left(\boldsymbol{y}_{m}\right)$ a sequence of $\boldsymbol{X}_{T}(\underline{\boldsymbol{\xi}} ; \Omega)$ such that, $\forall m,\left\|\boldsymbol{y}_{m}\right\|_{0, \Omega}=1$ and

$$
\left\|\operatorname{curl} \boldsymbol{y}_{m}\right\|_{0, \Omega}+\left\|\operatorname{div} \underline{\boldsymbol{\xi}} \boldsymbol{y}_{m}\right\|_{0, \Omega}+\sum_{1 \leq i \leq I}\left|\left\langle\underline{\boldsymbol{\xi}} \boldsymbol{y}_{m} \cdot \boldsymbol{n}, 1\right\rangle_{H^{1 / 2}\left(\Sigma_{i}\right)}\right| \leq \frac{1}{m+1} \text {. }
$$


Step 1. Consider the solution to the Neumann problem

$$
\left\{\begin{array}{l}
\text { Find } q_{m}^{0} \in H_{\mathrm{zmv}}^{1}(\Omega) \text { such that } \\
\left(\underline{\boldsymbol{\xi}} \nabla q_{m}^{0} \mid \nabla q\right)_{0, \Omega}=\left(\underline{\boldsymbol{\xi}} \boldsymbol{y}_{m} \mid \nabla q\right)_{0, \Omega}, \forall q \in H_{\mathrm{zmv}}^{1}(\Omega) .
\end{array}\right.
$$

The problem is well-posed by theorem 2.1. Taking $q=q_{m}^{0}$ and integrating by parts, one gets, as $\boldsymbol{y}_{m} \in \boldsymbol{H}_{0}(\operatorname{div} \underline{\boldsymbol{\xi}} ; \Omega)$ :

$$
\left|\left(\underline{\boldsymbol{\xi}} \nabla q_{m}^{0} \mid \nabla q_{m}^{0}\right)_{0, \Omega}\right|=\left|\left(-\operatorname{div} \underline{\boldsymbol{\xi}} \boldsymbol{y}_{m} \mid q_{m}^{0}\right)_{0, \Omega}\right| \leq\left\|\operatorname{div} \underline{\boldsymbol{\xi}} \boldsymbol{y}_{m}\right\|_{0, \Omega}\left\|q_{m}^{0}\right\|_{0, \Omega} .
$$

Using the relation (3) on the left-hand side, as well as the Poincaré-Wirtinger inequality on the right-hand side, leads to

$$
\left\|\nabla q_{m}^{0}\right\|_{0, \Omega} \lesssim\left\|\operatorname{div} \underline{\boldsymbol{\xi}} \boldsymbol{y}_{m}\right\|_{0, \Omega}
$$

so $\left\|\nabla q_{m}^{0}\right\|_{0, \Omega} \longrightarrow 0$.

Step 2. With the help of the second-kind Helmholtz decomposition (9) of $\boldsymbol{y}_{m}$, we define $\boldsymbol{x}_{m}:=$ $\boldsymbol{y}_{m}-\nabla q_{m}^{0} \in \boldsymbol{K}_{T}(\underline{\boldsymbol{\xi}} ; \Omega)$. Note that $\nabla q_{m}^{0} \in \boldsymbol{H}_{0}(\operatorname{div} \underline{\boldsymbol{\xi}} ; \Omega)$. Consider the finite-dimensional space

$$
Q_{T}(\underline{\boldsymbol{\xi}} ; \dot{\Omega}):=\left\{\dot{q} \in H_{\mathrm{zmv}}^{1}(\dot{\Omega}) \mid \operatorname{div} \underline{\boldsymbol{\xi}} \widetilde{\nabla \dot{q}}=0 \text { in } \Omega, \underline{\boldsymbol{\xi}} \widetilde{\nabla} \dot{q} \cdot \boldsymbol{n}_{\mid \partial \Omega}=0,[\dot{q}]_{\Sigma_{i}}=c s t_{i}, 1 \leq i \leq I\right\},
$$

where $c s t_{i}$ is a constant field on $\Sigma_{i}$. Let us introduce the problem

$$
\left\{\begin{array}{l}
\text { Find } \dot{q}_{m}^{\Sigma} \in Q_{T}(\underline{\boldsymbol{\xi}} ; \dot{\Omega}) \text { such that } \\
\left(\underline{\boldsymbol{\xi}} \nabla \dot{q}_{m}^{\Sigma} \mid \nabla \dot{q}\right)_{0, \dot{\Omega}}=\left(\underline{\boldsymbol{\xi}} \boldsymbol{x}_{m} \mid \nabla \dot{q}\right)_{0, \dot{\Omega}} \forall \dot{q} \in Q_{T}(\underline{\boldsymbol{\xi}} ; \dot{\Omega}) .
\end{array}\right.
$$

This problem is well-posed, adapting the proof of theorem 2.1, and using Poincaré-Wirtinger inequality in $H_{\mathrm{zmv}}^{1}(\dot{\Omega})$. Taking $\dot{q}=\dot{q}_{m}^{\Sigma}$ and using the integration by parts formula (65), one has, as $\operatorname{div} \boldsymbol{\xi} \boldsymbol{x}_{m}=0$,

$$
\left(\underline{\boldsymbol{\xi}} \nabla \dot{q}_{m}^{\Sigma} \mid \nabla \dot{q}_{m}^{\Sigma}\right)_{0, \dot{\Omega}}=\sum_{1 \leq i \leq I}\left\langle\underline{\boldsymbol{\xi}} \boldsymbol{x}_{m} \cdot \boldsymbol{n},\left[\dot{q}_{m}^{\Sigma}\right]_{\Sigma_{i}}\right\rangle_{H^{1 / 2}\left(\Sigma_{i}\right)}=\sum_{1 \leq i \leq I} \overline{\left[\dot{q}_{m}^{\Sigma}\right]_{\Sigma_{i}}}\left\langle\underline{\boldsymbol{\xi}} \boldsymbol{x}_{m} \cdot \boldsymbol{n}, 1\right\rangle_{H^{1 / 2}\left(\Sigma_{i}\right)} .
$$

As $Q_{T}(\underline{\xi} ; \dot{\Omega})$ is a finite-dimensional vector space, all the norms are equivalent, and among them, $\|\widetilde{\nabla \cdot}\|_{0, \Omega}$ and $\max _{i}\left|[\cdot]_{\Sigma_{i}}\right|$. Then, using additionally relation (3), there holds

$$
\left\|\dot{q}_{m}^{\Sigma}\right\|_{Q_{T}(\underline{\boldsymbol{\xi}} ; \dot{\Omega})}^{2} \lesssim\left(\left\|\dot{q}_{m}^{\Sigma}\right\|_{Q_{T}(\underline{\xi} ; \dot{\Omega})} \sum_{1 \leq i \leq I}\left|\left\langle\underline{\boldsymbol{\xi}} \boldsymbol{x}_{m} \cdot \boldsymbol{n}, 1\right\rangle_{H^{1 / 2}\left(\Sigma_{i}\right)}\right|\right) .
$$

In addition, $\left\langle\underline{\boldsymbol{\xi}} \boldsymbol{x}_{m} \cdot \boldsymbol{n}, 1\right\rangle_{H^{1 / 2}\left(\Sigma_{i}\right)}=\left\langle\underline{\boldsymbol{\xi}} \boldsymbol{y}_{m} \cdot \boldsymbol{n}, 1\right\rangle_{H^{1 / 2}\left(\Sigma_{i}\right)}-\left\langle\underline{\boldsymbol{\xi}} \nabla q_{m}^{0} \cdot \boldsymbol{n}, 1\right\rangle_{H^{1 / 2}\left(\Sigma_{i}\right)}$. For $1 \leq i \leq I$, let $\dot{q}_{i}$ be the unique element of $Q_{T}(\underline{\xi} ; \dot{\Omega})$ such that $\left[\dot{q}_{i}\right]_{\Sigma_{j}}=\delta_{i, j}$ for $1 \leq j \leq I$. If one recalls that $\nabla q_{m}^{0} \in \boldsymbol{H}_{0}(\operatorname{div} \underline{\boldsymbol{\xi}} ; \Omega)$, then there holds, using the integration by parts formula (65),

$$
\begin{aligned}
\left|\left\langle\underline{\boldsymbol{\xi}} \nabla q_{m}^{0} \cdot \boldsymbol{n}, 1\right\rangle_{H^{1 / 2}\left(\Sigma_{i}\right)}\right| & =\left|\sum_{1 \leq j \leq I}\left\langle\underline{\boldsymbol{\xi}} \nabla q_{m}^{0} \cdot \boldsymbol{n},\left[\dot{q}_{i}\right]_{\Sigma_{j}}\right\rangle_{H^{1 / 2}\left(\Sigma_{j}\right)}\right| \\
& =\left|\left(\underline{\boldsymbol{\xi}} \nabla q_{m}^{0} \mid \nabla \dot{q}_{i}\right)_{0, \dot{\Omega}}+\left(\operatorname{div} \underline{\boldsymbol{\xi}} \nabla q_{m}^{0} \mid \dot{q}_{i}\right)_{0, \dot{\Omega}}\right| \\
& \lesssim\left\|\nabla q_{m}^{0}\right\|_{0, \dot{\Omega}}\left\|\nabla \dot{q}_{i}\right\|_{0, \dot{\Omega}}+\left\|\operatorname{div} \underline{\boldsymbol{\xi}} \nabla q_{m}^{0}\right\|_{0, \dot{\Omega}}\left\|\dot{q}_{i}\right\|_{0, \dot{\Omega}} \\
& \lesssim\left\|\nabla q_{m}^{0}\right\|_{\boldsymbol{H}(\operatorname{div} \boldsymbol{\xi} ; \Omega)} \\
& \lesssim\left\|\operatorname{div} \underline{\boldsymbol{\xi}} \boldsymbol{y}_{m}\right\|_{0, \Omega},
\end{aligned}
$$


the latter because of (17) and $\operatorname{div} \underline{\boldsymbol{\xi}} \nabla q_{m}^{0}=\operatorname{div} \underline{\boldsymbol{\xi}} \boldsymbol{y}_{m}$ in $\Omega$. Hence,

$$
\left\|\dot{q}_{m}^{\Sigma}\right\|_{Q_{T}(\underline{\boldsymbol{\xi} ; \dot{\Omega})}} \lesssim\left(\sum_{1 \leq i \leq I}\left|\left\langle\underline{\boldsymbol{\xi}} \boldsymbol{y}_{m} \cdot \boldsymbol{n}, 1\right\rangle_{H^{1 / 2}\left(\Sigma_{i}\right)}\right|+\left\|\operatorname{div} \underline{\boldsymbol{\xi}} \boldsymbol{y}_{m}\right\|_{0, \Omega}\right)
$$

which implies that $\left\|\widetilde{\nabla \dot{q}_{m}^{\Sigma}}\right\|_{0, \Omega}=\left\|\dot{q}_{m}^{\Sigma}\right\|_{Q_{T}(\xi ; \dot{\Omega})} \longrightarrow 0$.

Step 3. Let $\boldsymbol{z}_{m}:=\boldsymbol{y}_{m}-\nabla q_{m}^{0}-\widetilde{\nabla \dot{q}_{m}^{\Sigma}} \in \boldsymbol{X}_{T}(\underline{\boldsymbol{\xi}}, \Omega)$. There holds $\operatorname{curl} \boldsymbol{z}_{m}=\operatorname{curl} \boldsymbol{y}_{m}, \operatorname{div} \underline{\boldsymbol{\xi}}_{m}=0$, and additionally $\left\langle\underline{\boldsymbol{\xi}} \boldsymbol{z}_{m} \cdot \boldsymbol{n}, 1\right\rangle_{H^{1 / 2}\left(\Sigma_{i}\right)}=0$ for $1 \leq i \leq I$. First, one has by using the integration by parts formula $(65)$

$$
\left\langle\underline{\boldsymbol{\xi}} \boldsymbol{z}_{m} \cdot \boldsymbol{n}, 1\right\rangle_{H^{1 / 2}\left(\Sigma_{i}\right)}=\sum_{1 \leq j \leq I}\left\langle\underline{\boldsymbol{\xi}} \boldsymbol{z}_{m} \cdot \boldsymbol{n},\left[\dot{q}_{i}\right]_{\Sigma_{j}}\right\rangle_{H^{1 / 2}\left(\Sigma_{j}\right)}=\left(\underline{\boldsymbol{\xi}} \boldsymbol{z}_{m} \mid \nabla \dot{q}_{i}\right)_{0, \dot{\Omega}}+\left(\operatorname{div} \underline{\boldsymbol{\xi}} \boldsymbol{z}_{m} \mid \dot{q}_{i}\right)_{0, \dot{\Omega}} \cdot
$$

Then, $\left(\underline{\boldsymbol{\xi}} \boldsymbol{z}_{m} \mid \nabla \dot{q}_{i}\right)_{0, \dot{\Omega}}=\left(\underline{\boldsymbol{\xi}} \boldsymbol{x}_{m} \mid \nabla \dot{q}_{i}\right)_{0, \dot{\Omega}}-\left(\underline{\boldsymbol{\xi}} \nabla \dot{q}_{m}^{\Sigma} \mid \nabla \dot{q}_{i}\right)_{0, \dot{\Omega}}=0$ by definition of $\dot{q}_{m}^{\Sigma}$, while div $\underline{\boldsymbol{\xi}} \boldsymbol{z}_{m}=$ 0 by definition of $\boldsymbol{z}_{m}$.

One has also $\boldsymbol{z}_{m} \in \boldsymbol{H}_{0}(\operatorname{div} \boldsymbol{\xi} 0 ; \Omega)$ so, according to the vector potential Theorem 3.5.1 [5]: there exists $\boldsymbol{w}_{m} \in \boldsymbol{X}_{N}(\Omega)$ such that $\underline{\boldsymbol{\xi}} \boldsymbol{z}_{m}=\operatorname{curl} \boldsymbol{w}_{m}$ and $\operatorname{div} \boldsymbol{w}_{m}=0$ in $\Omega$, and $\left\|\boldsymbol{w}_{m}\right\|_{\boldsymbol{H}(\operatorname{curl} ; \Omega)} \lesssim$ $\left\|\boldsymbol{\xi}_{m}\right\|_{0, \Omega} ;$ in particular, $\left\|\boldsymbol{w}_{m}\right\|_{\boldsymbol{H}(\mathbf{c u r l} ; \Omega)} \lesssim\left\|\boldsymbol{z}_{m}\right\|_{0, \Omega}$.

We have by integration by parts $\left(\boldsymbol{z}_{m} \mid \underline{\boldsymbol{\xi}} \boldsymbol{z}_{m}\right)_{0, \Omega}=\left(\boldsymbol{z}_{m} \mid \operatorname{curl} \boldsymbol{w}_{m}\right)_{0, \Omega}=\left(\operatorname{curl} \boldsymbol{z}_{m} \mid \boldsymbol{w}_{m}\right)_{0, \Omega}=$ $\left(\operatorname{curl} \boldsymbol{y}_{m} \mid \boldsymbol{w}_{m}\right)_{0, \Omega}$. Using again the relation (3), we find

$$
\left\|\boldsymbol{z}_{m}\right\|_{0, \Omega}^{2} \lesssim\left\|\operatorname{curl} \boldsymbol{y}_{m}\right\|_{0, \Omega}\left\|\boldsymbol{w}_{m}\right\|_{0, \Omega} \lesssim\left\|\operatorname{curl} \boldsymbol{y}_{m}\right\|_{0, \Omega}\left\|\boldsymbol{z}_{m}\right\|_{0, \Omega}
$$

so that $\left\|\boldsymbol{z}_{m}\right\|_{0, \Omega} \lesssim\left\|\operatorname{curl} \boldsymbol{y}_{m}\right\|_{0, \Omega}$. It follows that $\left\|\boldsymbol{z}_{m}\right\|_{0, \Omega} \longrightarrow 0$.

As $\boldsymbol{y}_{m}=\boldsymbol{z}_{m}+\nabla q_{m}^{0}+\widetilde{\nabla \dot{q}_{m}^{\Sigma}}$, we conclude that $\left\|\boldsymbol{y}_{m}\right\|_{0, \Omega} \longrightarrow 0$, but that contradicts $\left\|\boldsymbol{y}_{m}\right\|_{0, \Omega}=1$ for all $m$.

One can also extend the compact embedding result of Theorem 7.5.3 in Ref. [5].

Theorem 2.7. Assume that (Top) I holds. Under assumption (2), the embedding of $\boldsymbol{X}_{T}(\underline{\boldsymbol{\xi}} ; \Omega)$ into $\boldsymbol{L}^{2}(\Omega)$ is compact.

Proof. Let $\left(\boldsymbol{y}_{m}\right)$ be a bounded sequence of $\boldsymbol{X}_{T}(\underline{\boldsymbol{\xi}} ; \Omega)$. As in the previous proof (Steps 1., 2., 3.), we introduce $q_{m}^{0} \in H_{\mathrm{zmv}}^{1}(\Omega), \dot{q}_{m}^{\Sigma} \in Q_{T}(\underline{\boldsymbol{\xi}} ; \dot{\Omega})$, and $\boldsymbol{w}_{m} \in \boldsymbol{X}_{N}(\Omega)$, such that $\boldsymbol{y}_{m}=$ $\underline{\boldsymbol{\xi}}^{-1} \operatorname{curl} \boldsymbol{w}_{m}+\nabla q_{m}^{0}+\widetilde{\nabla \dot{q}_{m}^{\Sigma}}$. Additionally, there holds (see previous proof):

$$
\begin{aligned}
\left\|\nabla q_{m}^{0}\right\|_{0, \Omega} & \lesssim\left\|\operatorname{div} \underline{\boldsymbol{\xi}} \boldsymbol{y}_{m}\right\|_{0, \Omega} ; \\
\left\|\widetilde{\nabla \dot{q}_{m}^{\Sigma}}\right\|_{0, \Omega} & \lesssim\left(\left\|\operatorname{div} \underline{\boldsymbol{\xi}} \boldsymbol{y}_{m}\right\|_{0, \Omega}+\sum_{i}\left|\left\langle\underline{\boldsymbol{\xi}} \boldsymbol{y}_{m} \cdot \boldsymbol{n}, 1\right\rangle_{H^{1 / 2}\left(\Sigma_{i}\right)}\right|\right) \\
\left\|\boldsymbol{w}_{m}\right\|_{\boldsymbol{H}(\mathbf{c u r l} ; \Omega)} & \lesssim\left\|\operatorname{curl} \boldsymbol{y}_{m}\right\|_{0, \Omega} .
\end{aligned}
$$

Let us begin with $\left(\dot{q}_{m}^{\Sigma}\right)$ : it is a bounded sequence of the finite-dimensional vector space $Q_{T}(\underline{\xi} ; \dot{\Omega})$, so it admits a converging subsequence (in particular in $\|\cdot\|_{1, \Omega}$-norm).

In addition, $\left(q_{m}^{0}\right)$ is a bounded sequence of $H^{1}(\Omega)$ so, by Rellich's theorem, it admits a converging susbsequence (denoted with the same index) in $L^{2}(\Omega)$. Similarly, $\boldsymbol{w}_{m}$ is a bounded sequence of $\boldsymbol{X}_{N}(\Omega)$, so by theorem 2.5 , it admits a converging subsequence in $\boldsymbol{L}^{2}(\Omega)$. It remains to prove that the subsequences $\left(\nabla q_{m}^{0}\right)$ and $\left(\operatorname{curl} \boldsymbol{w}_{m}\right)$ converge in $\boldsymbol{L}^{2}(\Omega)$.

By definition of $q_{m}^{0}$, for any $q$ in $H_{0}^{1}(\Omega)$, there holds by integration by parts

$$
\left(\underline{\boldsymbol{\xi}} \nabla q_{m n}^{0} \mid \nabla q\right)_{0, \Omega}=\left(\underline{\boldsymbol{\xi}} \boldsymbol{y}_{m n} \mid \nabla q\right)_{0, \Omega}=-\left(\operatorname{div} \underline{\boldsymbol{\xi}} \boldsymbol{y}_{m n} \mid q\right)_{0, \Omega} .
$$


Taking $q=q_{m n}^{0}$, one gets by property (2)

$$
\left\|\nabla q_{m n}^{0}\right\|_{0, \Omega}^{2} \lesssim\left\|\operatorname{div} \underline{\boldsymbol{\xi}} \boldsymbol{y}_{m n}\right\|_{0, \Omega}\left\|q_{m n}^{0}\right\|_{0, \Omega} \leq 2 \sup _{m}\left(\left\|\operatorname{div} \underline{\boldsymbol{\xi}} \boldsymbol{y}_{m}\right\|_{0, \Omega}\right)\left\|q_{m n}^{0}\right\|_{0, \Omega}
$$

Thus $\left(\nabla q_{m}^{0}\right)$ is a Cauchy sequence of $\boldsymbol{L}^{2}(\Omega)$, and hence it converges in this Hilbert space. By integration by parts (recall that $\boldsymbol{w}_{m n} \in \boldsymbol{X}_{N}(\Omega)$ ),

$$
\left(\underline{\boldsymbol{\xi}}^{-1} \operatorname{curl} \boldsymbol{w}_{m n} \mid \operatorname{curl} \boldsymbol{w}_{m n}\right)_{0, \Omega}=\left(\operatorname{curl}\left(\underline{\boldsymbol{\xi}}^{-1} \operatorname{curl} \boldsymbol{w}_{m n}\right) \mid \boldsymbol{w}_{m n}\right)_{0, \Omega}=\left(\operatorname{curl} \boldsymbol{y}_{m n} \mid \boldsymbol{w}_{m n}\right)_{0, \Omega} .
$$

As $\underline{\boldsymbol{\xi}}^{-1}$ satisfies the ellipticity condition (proposition 2.1), we find

$$
\left\|\operatorname{curl} \boldsymbol{w}_{m n}\right\|_{0, \Omega}^{2} \lesssim\left\|\operatorname{curl} \boldsymbol{y}_{m n}\right\|_{0, \Omega}\left\|\boldsymbol{w}_{m n}\right\|_{0, \Omega} \leq 2 \sup _{m}\left(\left\|\operatorname{curl} \boldsymbol{y}_{m}\right\|_{0, \Omega}\right)\left\|\boldsymbol{w}_{m n}\right\|_{0, \Omega},
$$

which proves that ( $\left.\operatorname{curl} \boldsymbol{w}_{m}\right)$ is also a Cauchy and hence converging sequence of $\boldsymbol{L}^{2}(\Omega)$.

As $\boldsymbol{y}_{m}=\underline{\boldsymbol{\xi}}^{-1} \operatorname{curl} \boldsymbol{w}_{m}+\nabla q_{m}^{0}+\widetilde{\nabla \dot{q}_{m}^{\Sigma}}$, the subsequence $\left(\boldsymbol{y}_{m}\right)$ converges in $\boldsymbol{L}^{2}(\Omega)$.

\section{Analysis of the Dirichlet problem}

In this section, we supplement the time-harmonic Maxwell's equation by a Dirichlet boundary condition on $\partial \Omega$ :

$$
\left\{\begin{array}{l}
\text { Find } \boldsymbol{E} \in \boldsymbol{H}(\mathbf{c u r l} ; \Omega) \text { such that } \\
\operatorname{curl}\left(\underline{\boldsymbol{\mu}}^{-1} \operatorname{curl} \boldsymbol{E}\right)-\omega^{2} \underline{\boldsymbol{\varepsilon}} \boldsymbol{E}=\boldsymbol{f} \text { in } \Omega \\
\boldsymbol{E} \times \boldsymbol{\boldsymbol { n }}=\boldsymbol{g} \text { on } \partial \Omega
\end{array}\right.
$$

where $\boldsymbol{g}$ is a boundary data.

We assume that the tensors $\underline{\boldsymbol{\varepsilon}}, \underline{\boldsymbol{\mu}} \in \underline{\mathbf{L}}^{\infty}(\Omega)$ are elliptic. The corresponding coercivity directions (or, equivalently, the parameters $\theta_{\epsilon}$ and $\theta_{\mu}$ in definition 2.1) may be different. We also assume that the volume data $\boldsymbol{f}$ belongs to $\boldsymbol{L}^{2}(\Omega)$, and that the surface data $\boldsymbol{g}$ is the tangential trace of a field $\boldsymbol{E}_{d} \in \boldsymbol{H}(\operatorname{curl} ; \Omega)$, that is $\boldsymbol{g}=\boldsymbol{E}_{d} \times \boldsymbol{n}_{\mid \partial \Omega}$. Further assumptions will be made to obtain the extra-regularity results.

\subsection{Variational formulation and well-posedness}

Let us derive the variational formulation of problem (20). In order to deal with a problem with homogeneous boundary condition, we introduce the new unknown $\boldsymbol{E}_{0}:=\boldsymbol{E}-\boldsymbol{E}_{d}$. It belongs to $\boldsymbol{H}_{0}(\mathbf{c u r l} ; \Omega)$ and, additionally, satisfies

$$
\operatorname{curl}\left(\underline{\boldsymbol{\mu}}^{-1} \operatorname{curl} \boldsymbol{E}_{0}\right)-\omega^{2} \underline{\varepsilon} \boldsymbol{E}_{0}=\boldsymbol{f}-\operatorname{curl}\left(\underline{\boldsymbol{\mu}}^{-1} \operatorname{curl} \boldsymbol{E}_{d}\right)+\omega^{2} \underline{\varepsilon} \boldsymbol{E}_{d} \quad \text { in } \Omega .
$$

By standard techniques, we get the equivalent variational formulation for $\boldsymbol{E}_{0}$ :

$$
\left\{\begin{array}{l}
\text { Find } \boldsymbol{E}_{0} \in \boldsymbol{H}_{0}(\operatorname{curl} ; \Omega) \text { such that } \\
\left(\underline{\boldsymbol{\mu}}^{-1} \operatorname{curl} \boldsymbol{E}_{0} \mid \operatorname{curl} \boldsymbol{F}\right)_{0, \Omega}-\omega^{2}\left(\underline{\varepsilon} \boldsymbol{E}_{0} \mid \boldsymbol{F}\right)_{0, \Omega}=\ell_{\mathrm{D}, 0}(\boldsymbol{F}), \forall \boldsymbol{F} \in \boldsymbol{H}_{0}(\operatorname{curl} ; \Omega) .
\end{array}\right.
$$

Above, $\ell_{\mathrm{D}, 0}: \boldsymbol{F} \mapsto\left(\boldsymbol{f}+\omega^{2} \underline{\boldsymbol{\varepsilon}} \boldsymbol{E}_{d} \mid \boldsymbol{F}\right)_{0, \Omega}-\left(\underline{\boldsymbol{\mu}}^{-1} \operatorname{curl} \boldsymbol{E}_{d} \mid \operatorname{curl} \boldsymbol{F}\right)_{0, \Omega}$ belongs to $\left(\boldsymbol{H}_{0}(\operatorname{curl} ; \Omega)\right)^{\prime}$. In addition, we observe that

$$
\left\|\ell_{\mathrm{D}, 0}\right\|_{\left(\boldsymbol{H}_{0}(\operatorname{curl} ; \Omega)\right)^{\prime}} \lesssim\|\boldsymbol{f}\|_{0, \Omega}+\left\|\boldsymbol{E}_{d}\right\|_{\boldsymbol{H}(\mathbf{c u r l} ; \Omega)}
$$


Remark 3.1. In terms of the total field $\boldsymbol{E}$, the variational formulation to be solved is

$$
\left\{\begin{array}{l}
\text { Find } \boldsymbol{E} \in \boldsymbol{H}(\operatorname{curl} ; \Omega) \text { such that } \\
\left(\underline{\boldsymbol{\mu}}^{-1} \operatorname{curl} \boldsymbol{E} \mid \operatorname{curl} \boldsymbol{F}\right)_{0, \Omega}-\omega^{2}(\underline{\varepsilon} \boldsymbol{E} \mid \boldsymbol{F})_{0, \Omega}=\ell_{\mathrm{D}}(\boldsymbol{F}), \forall \boldsymbol{F} \in \boldsymbol{H}_{0}(\operatorname{curl} ; \Omega), \\
\boldsymbol{E} \times \boldsymbol{n}=\boldsymbol{g} \text { on } \partial \Omega,
\end{array}\right.
$$

where $\ell_{\mathrm{D}}: \boldsymbol{F} \mapsto(\boldsymbol{f} \mid \boldsymbol{F})_{0, \Omega}$ belongs to $\left(\boldsymbol{H}_{0}(\mathbf{c u r l} ; \Omega)\right)^{\prime}$. This formulation can be used to solve the nonhomogeneous Dirichlet problem numerically, see section 5.

To study the well-posedness of the formulation (21), it is useful to introduce an equivalent problem with the help of the previously derived first-kind Helmholtz decomposition (7).

Lemma 3.1. The formulation (21) can be equivalently recast as follows: set $\boldsymbol{E}_{0}=\tilde{\boldsymbol{E}}+\nabla p$, with $p \in H_{0}^{1}(\Omega)$ and $\tilde{\boldsymbol{E}} \in \boldsymbol{K}_{N}(\underline{\varepsilon} ; \Omega)$, respectively governed by

$$
\left\{\begin{array}{l}
\text { Find } p \in H_{0}^{1}(\Omega) \text { such that } \\
-\omega^{2}(\underline{\varepsilon} \nabla p \mid \nabla q)_{0, \Omega}=\ell_{\mathrm{D}, 0}(\nabla q)_{0, \Omega}, \forall q \in H_{0}^{1}(\Omega)
\end{array}\right.
$$

and

$$
\left\{\begin{array}{l}
\text { Find } \tilde{\boldsymbol{E}} \in \boldsymbol{K}_{N}(\underline{\varepsilon} ; \Omega) \text { such that } \\
\left(\underline{\boldsymbol{\mu}}^{-1} \operatorname{curl} \tilde{\boldsymbol{E}} \mid \operatorname{curl} \tilde{\boldsymbol{F}}\right)_{0, \Omega}-\omega^{2}(\underline{\varepsilon} \tilde{\boldsymbol{E}} \mid \tilde{\boldsymbol{F}})_{0, \Omega}=\omega^{2}(\underline{\varepsilon} \nabla p \mid \tilde{\boldsymbol{F}})_{0, \Omega}+\ell_{\mathrm{D}, 0}(\tilde{\boldsymbol{F}}), \forall \tilde{\boldsymbol{F}} \in \boldsymbol{K}_{N}(\underline{\varepsilon} ; \Omega) .
\end{array}\right.
$$

Proof. Direct. Let us introduce the first-kind Helmholtz decomposition (7) of $\boldsymbol{E}_{0}: \boldsymbol{E}_{0}=\tilde{\boldsymbol{E}}+\nabla p$, with $p \in H_{0}^{1}(\Omega)$ and $\tilde{\boldsymbol{E}} \in \boldsymbol{K}_{N}(\underline{\varepsilon} ; \Omega)$. Taking $\boldsymbol{F}=\nabla q$ for any $q \in H_{0}^{1}(\Omega)$ in (21), we get

$$
-\omega^{2}(\underline{\varepsilon}(\tilde{\boldsymbol{E}}+\nabla p) \mid \nabla q)_{0, \Omega}=\ell_{\mathrm{D}, 0}(\nabla q)
$$

and, since $\tilde{\boldsymbol{E}} \in \boldsymbol{K}_{N}(\underline{\varepsilon} ; \Omega)$, it holds that, $\forall q \in \boldsymbol{H}_{0}^{1}(\Omega),(\underline{\varepsilon} \tilde{\boldsymbol{E}} \mid \nabla q)_{0, \Omega}=0$, so $p$ is governed by (24).

On the other hand, for $\tilde{\boldsymbol{E}}=\boldsymbol{E}_{0}-\nabla p$, one has

$$
\left(\underline{\boldsymbol{\mu}}^{-1} \operatorname{curl} \tilde{\boldsymbol{E}} \mid \operatorname{curl} \boldsymbol{F}\right)_{0, \Omega}-\omega^{2}(\underline{\varepsilon} \tilde{\boldsymbol{E}} \mid \boldsymbol{F})_{0, \Omega}=\omega^{2}(\underline{\varepsilon} \nabla p \mid \boldsymbol{F})_{0, \Omega}+\ell_{\mathrm{D}, 0}(\boldsymbol{F})
$$

for any $\boldsymbol{F} \in \boldsymbol{H}_{0}(\operatorname{curl} ; \Omega)$, hence in particular for any $\tilde{\boldsymbol{F}} \in \boldsymbol{K}_{N}(\underline{\varepsilon} ; \Omega)$ : $\tilde{\boldsymbol{E}}$ is governed by (25). Reverse. By summation one has, $\forall q \in H_{0}^{1}(\Omega), \forall \tilde{\boldsymbol{F}} \in \boldsymbol{K}_{N}(\underline{\varepsilon} ; \Omega)$ :

$$
\begin{aligned}
\left(\underline{\boldsymbol{\mu}}^{-1} \operatorname{curl} \tilde{\boldsymbol{E}} \mid \operatorname{curl} \tilde{\boldsymbol{F}}\right)_{0, \Omega}-\omega^{2}(\underline{\varepsilon} \tilde{\boldsymbol{E}} \mid \tilde{\boldsymbol{F}})_{0, \Omega}-\omega^{2}(\underline{\varepsilon} \nabla p \mid \nabla q)_{0, \Omega} & \\
& =\omega^{2}(\underline{\varepsilon} \nabla p \mid \tilde{\boldsymbol{F}})_{0, \Omega}+\ell_{\mathrm{D}, 0}(\tilde{\boldsymbol{F}})+\ell_{\mathrm{D}, 0}(\nabla q) .
\end{aligned}
$$

One can add the vanishing terms $\left(\underline{\boldsymbol{\mu}}^{-1} \operatorname{curl} \nabla p \mid \operatorname{curl} \tilde{\boldsymbol{F}}\right)_{0, \Omega},\left(\underline{\boldsymbol{\mu}}^{-1} \operatorname{curl}(\tilde{\boldsymbol{E}}+\nabla p) \mid \operatorname{curl} \nabla q\right)_{0, \Omega}$ and $-\omega^{2}(\underline{\varepsilon} \tilde{\boldsymbol{E}} \mid \nabla q)_{0, \Omega}$ to the left-hand side. Introducing $\boldsymbol{E}_{0}:=\tilde{\boldsymbol{E}}+\nabla p \in \boldsymbol{H}_{0}(\mathbf{c u r l} ; \Omega)$, one gets, after simple rearrangements:

$$
\left(\underline{\boldsymbol{\mu}}^{-1} \operatorname{curl} \boldsymbol{E}_{0} \mid \operatorname{curl}(\tilde{\boldsymbol{F}}+\nabla q)\right)_{0, \Omega}-\omega^{2}\left(\underline{\varepsilon} \boldsymbol{E}_{0} \mid \tilde{\boldsymbol{F}}+\nabla q\right)_{0, \Omega}=\ell_{\mathrm{D}, 0}(\tilde{\boldsymbol{F}}+\nabla q) .
$$

Finally, as $\tilde{\boldsymbol{F}}$ and $q$ span respectively $\boldsymbol{K}_{N}(\underline{\varepsilon} ; \Omega)$ and $H_{0}^{1}(\Omega)$, the sum $\tilde{\boldsymbol{F}}+\nabla q$ spans the whole $\boldsymbol{H}_{0}(\operatorname{curl} ; \Omega)$, thanks to (7): $\boldsymbol{E}_{0}$ is governed by (21). 
Remark 3.2. The term $\omega^{2}(\underline{\varepsilon} \nabla p \mid \tilde{\boldsymbol{F}})_{0, \Omega}$ in formulation (25) vanishes automatically only if $\underline{\boldsymbol{\varepsilon}}$ is a Hermitian tensor field.

Then, we have the following results.

Lemma 3.2. The formulation (24) is well-posed. Moreover, one has the bound $\|p\|_{1, \Omega} \lesssim$ $\|\boldsymbol{f}\|_{0, \Omega}+\left\|\boldsymbol{E}_{d}\right\|_{0, \Omega}$.

Proof. Well-posedness is an immediate consequence of theorem 2.1. Since $\ell_{\mathrm{D}, 0} \in\left(\boldsymbol{H}_{0}(\mathbf{c u r l} ; \Omega)\right)^{\prime}$, and $\nabla$ is a continuous mapping from $H_{0}^{1}(\Omega)$ to $\boldsymbol{H}_{0}(\operatorname{curl} ; \Omega)$, one has $\ell_{\mathrm{D}, 0} \circ \nabla \in\left(H_{0}^{1}(\Omega)\right)^{\prime}$. The bound on $\|p\|_{1, \Omega}$ is a straightforward consequence of the expression $\ell_{\mathrm{D}, 0}(\nabla q)=(\boldsymbol{f}+$ $\left.\omega^{2} \underline{\varepsilon} \boldsymbol{E}_{d} \mid \nabla q\right)_{0, \Omega}$.

Recall that $\boldsymbol{K}_{N}(\underline{\varepsilon} ; \Omega)$ is equipped with the norm $\|\cdot\|_{\boldsymbol{H}(\mathbf{c u r l} ; \Omega)}$. Thanks to the compact embedding of $\boldsymbol{K}_{N}(\underline{\varepsilon} ; \Omega)$ into $\boldsymbol{L}^{2}(\Omega)$ (see Theorem 2.5), the formulation (25) enters Fredholm's alternative (see e.g. [5]).

Lemma 3.3. The formulation (25) enters Fredholm's alternative:

- either the problem (25) admits a unique solution $\tilde{\boldsymbol{E}}$ in $\boldsymbol{K}_{N}(\underline{\varepsilon} ; \Omega)$, which depends continuously on the data $\boldsymbol{f}$ and $\boldsymbol{E}_{d}$ :

$$
\|\tilde{\boldsymbol{E}}\|_{\boldsymbol{H}(\mathbf{c u r l} ; \Omega)} \lesssim\|\boldsymbol{f}\|_{0, \Omega}+\left\|\boldsymbol{E}_{d}\right\|_{\boldsymbol{H}(\operatorname{curl} ; \Omega)} ;
$$

- or, the problem (25) has solutions if, and only if, $\boldsymbol{f}$ and $\boldsymbol{E}_{d}$ satisfy a finite number of compatibility conditions; in this case, the space of solutions is an affine space of finite dimension. Additionally, the component of the solution which is orthogonal (in the sense of the $\boldsymbol{H}_{0}(\mathbf{c u r l} ; \Omega)$ scalar product) to the corresponding linear vector space, depends continuously on the data $\boldsymbol{f}$ and $\boldsymbol{E}_{d}$.

Proof. Let us split the left-hand side of (25) in two terms. Let $\alpha>0$, recalling that $\boldsymbol{\mu}^{-1}$ satisfies assumption (2), and using the notations of proposition 2.1, we introduce two sesquilinear forms, namely $a:(\boldsymbol{u}, \boldsymbol{v}) \mapsto\left(\underline{\boldsymbol{\mu}}^{-1} \operatorname{curl} \boldsymbol{u} \mid \operatorname{curl} \boldsymbol{v}\right)_{0, \Omega}+\alpha \mathrm{e}^{\mathrm{i} \theta_{\mu}}(\boldsymbol{u} \mid \boldsymbol{v})_{0, \Omega}$, and $b:(\boldsymbol{u}, \boldsymbol{v}) \mapsto\left(\underline{\boldsymbol{\varepsilon}}^{\prime} \boldsymbol{u} \mid \boldsymbol{v}\right)_{0, \Omega}$ with $\underline{\varepsilon}^{\prime}:=-\omega^{2} \underline{\varepsilon}-\alpha \mathrm{e}^{\mathrm{i} \theta_{\mu}} \underline{\mathbf{1}} \in \underline{\mathbf{L}}^{\infty}(\Omega)$.

We claim that the form $a$ is coercive on $\boldsymbol{K}_{N}(\underline{\varepsilon} ; \Omega)$. Indeed:

$$
\begin{aligned}
|a(\boldsymbol{v}, \boldsymbol{v})| & =\left|\left(\underline{\boldsymbol{\mu}}^{-1} \operatorname{curl} \boldsymbol{v} \mid \operatorname{curl} \boldsymbol{v}\right)_{0, \Omega}+\alpha \mathrm{e}^{\mathrm{i} \theta_{\mu}}(\boldsymbol{v} \mid \boldsymbol{v})_{0, \Omega}\right| \\
& \geq \Re\left[\mathrm{e}^{-\mathrm{i} \theta_{\mu}}\left(\underline{\boldsymbol{\mu}}^{-1} \operatorname{curl} \boldsymbol{v} \mid \operatorname{curl} \boldsymbol{v}\right)_{0, \Omega}+\alpha(\boldsymbol{v} \mid \boldsymbol{v})_{0, \Omega}\right] \\
& \geq\left(\mu^{-1}\right)_{-}\|\operatorname{curl} \boldsymbol{v}\|_{0, \Omega}^{2}+\alpha\|\boldsymbol{v}\|_{0, \Omega}^{2} \\
& \gtrsim\|\boldsymbol{v}\|_{\boldsymbol{H}(\operatorname{curl} ; \Omega)}^{2} .
\end{aligned}
$$

In addition, $|b(\boldsymbol{u}, \boldsymbol{v})| \leq\left\|\underline{\boldsymbol{\varepsilon}}^{\prime}\right\|_{\underline{\mathbf{L}}^{\infty}(\Omega)}\|\boldsymbol{u}\|_{0, \Omega}\|\boldsymbol{v}\|_{0, \Omega} \lesssim\|\boldsymbol{u}\|_{0, \Omega}\|\boldsymbol{v}\|_{\boldsymbol{K}_{N}(\underline{\varepsilon} ; \Omega)}$, so the form $b$ is continuous on $\boldsymbol{L}^{2}(\Omega) \times \boldsymbol{K}_{N}(\underline{\varepsilon} ; \Omega)$.

The embedding of $\boldsymbol{K}_{N}(\underline{\varepsilon} ; \Omega)$ into $\boldsymbol{L}^{2}(\Omega)$ is compact by theorem 2.5. Hence problem (25) enters the coercive + compact framework, and then Fredholm's alternative.

Regarding finally the bound, one uses simply the bounds on $\|p\|_{1, \Omega}$ (see lemma 3.2) and on $\left\|\ell_{\mathrm{D}, 0}\right\|_{\left(\boldsymbol{H}_{0}(\mathbf{c u r l} ; \Omega)\right)^{\prime}}($ see $(22))$.

We are now in a position to solve formulation (21) by regrouping the previous results. 
Theorem 3.1. The formulation (21) with unknown $\boldsymbol{E}_{0}$ enters Fredholm's alternative:

- either the problem (21) admits a unique solution $\boldsymbol{E}_{0}$ in $\boldsymbol{H}_{0}(\mathbf{c u r l} ; \Omega)$, which depends continuously on the data $\boldsymbol{f}$ and $\boldsymbol{E}_{d}$ :

$$
\left\|\boldsymbol{E}_{0}\right\|_{\boldsymbol{H}(\operatorname{curl} ; \Omega)} \lesssim\|\boldsymbol{f}\|_{0, \Omega}+\left\|\boldsymbol{E}_{d}\right\|_{\boldsymbol{H}(\operatorname{curl} ; \Omega)}
$$

- or, the problem (21) has solutions if, and only if, $\boldsymbol{f}$ and $\boldsymbol{E}_{d}$ satisfy a finite number of compatibility conditions; in this case, the space of solutions is an affine space of finite dimension. Additionally, the component of the solution which is orthogonal (in the sense of the $\boldsymbol{H}_{0}(\mathbf{c u r l} ; \Omega)$ scalar product) to the corresponding linear vector space, depends continuously on the data $\boldsymbol{f}$ and $\boldsymbol{E}_{d}$.

Moreover, each alternative occurs simultaneously for formulations (25) and (21).

Assumption. In the rest of the manuscript, we assume the problem (25) has a unique solution, such that the problem (21) is well-posed and, in particular, the estimate (26) holds.

\subsection{Extra-regularity of the solution}

The next two subsections aim at determining the extra-regularity of the solution and the solution's curl, depending on the extra-regularity of the data. We make the following extra-regularity assumptions for the next two subsections. We assume that $\partial \Omega$ is of class $\mathcal{C}^{2}$ and that $\boldsymbol{\mu}, \underline{\varepsilon} \in$ $\underline{\mathbf{C}}^{1}(\bar{\Omega})$. Regarding the extra-regularity of $\boldsymbol{f}$ and $\boldsymbol{E}_{d}$, we assume div $\boldsymbol{f} \in H^{\mathbf{s}-1}(\Omega)=\left(H_{0}^{1-\overline{\mathbf{s}}}(\Omega)\right)^{\prime}$, $\boldsymbol{E}_{d} \in \boldsymbol{H}^{\mathrm{r}}(\Omega)$ and $\operatorname{curl} \boldsymbol{E}_{d} \in \boldsymbol{H}^{\mathbf{r}^{\prime}}(\Omega)$ for given $\mathbf{s}, \mathbf{r}, \mathbf{r}^{\prime}$ in $[0,1] \backslash\left\{\frac{1}{2}\right\}$.

Let us recall a result on the continuous splitting of fields of $\boldsymbol{H}_{0}(\operatorname{curl} ; \Omega)$, cf. Lemma 2.4 in Ref. [21], or Theorem 3.6.7 in Ref. [5]:

Theorem 3.2. Let $\Omega$ be a domain. For all $\boldsymbol{u}$ in $\boldsymbol{H}_{0}(\operatorname{curl} ; \Omega)$, there exist $\boldsymbol{u}^{\text {reg }}$ in $\boldsymbol{H}^{1}(\Omega)$ and $\phi$ in $H_{0}^{1}(\Omega)$, such that

$$
\boldsymbol{u}=\boldsymbol{u}^{\mathrm{reg}}+\nabla \phi \text { in } \Omega, \text { with }\left\|\boldsymbol{u}^{\mathrm{reg}}\right\|_{1, \Omega}+\|\phi\|_{1, \Omega} \lesssim\|\boldsymbol{u}\|_{\boldsymbol{H}(\mathbf{c u r l} ; \Omega)} .
$$

With theorem 3.2 at hand, we introduce the splitting of $\boldsymbol{E}_{0}$ :

$$
\left\{\begin{array}{l}
\boldsymbol{E}_{0}=\boldsymbol{E}^{\mathrm{reg}}+\nabla \phi_{E}, \text { with } \boldsymbol{E}^{\mathrm{reg}} \in \boldsymbol{H}^{1}(\Omega), \phi_{E} \in H_{0}^{1}(\Omega), \text { and } \\
\left\|\boldsymbol{E}^{\mathrm{reg}}\right\|_{1, \Omega}+\left\|\phi_{E}\right\|_{1, \Omega} \lesssim\left\|\boldsymbol{E}_{0}\right\|_{\boldsymbol{H}(\mathbf{c u r l} ; \Omega)}
\end{array}\right.
$$

Taking $\boldsymbol{F}=\nabla q$ in $(21)$ for any $q \in H_{0}^{1}(\Omega)$, it holds that $-\omega^{2}\left(\underline{\boldsymbol{\varepsilon}} \boldsymbol{E}_{0} \mid \nabla q\right)_{0, \Omega}=\left(\boldsymbol{f}+\omega^{2} \underline{\varepsilon} \boldsymbol{E}_{d} \mid \nabla q\right)_{0, \Omega}$. As a consequence, $\phi_{E}$ is governed by the Dirichlet problem

$$
\left\{\begin{array}{l}
\text { Find } \phi_{E} \in H_{0}^{1}(\Omega) \text { such that } \\
\omega^{2}\left(\underline{\varepsilon} \nabla \phi_{E} \mid \nabla q\right)_{0, \Omega}=\left(\operatorname{div} \boldsymbol{f}+\omega^{2} \operatorname{div} \underline{\boldsymbol{\varepsilon}} \boldsymbol{E}_{d}+\omega^{2} \operatorname{div} \underline{\boldsymbol{\varepsilon}} \boldsymbol{E}^{\mathrm{reg}} \mid q\right)_{0, \Omega}, \forall q \in H_{0}^{1}(\Omega) .
\end{array}\right.
$$

Let us recall the fundamental regularity result for solutions of the Dirichlet problem, see Theorem 3.4.5 in Ref. [14]:

Theorem 3.3 (Shift theorem). Let $\Omega$ be a bounded domain of boundary $\partial \Omega$, $\ell$ in $\left(H_{0}^{1}(\Omega)\right)^{\prime}$, and $p$ governed by

$$
\left\{\begin{array}{l}
\text { Find } p \in H_{0}^{1}(\Omega) \text { such that } \\
(\underline{\boldsymbol{\xi}} \nabla p \mid \nabla q)_{0, \Omega}=\ell(q), \forall q \in H_{0}^{1}(\Omega) .
\end{array}\right.
$$


If the tensor coefficient $\boldsymbol{\xi}$ fulfills the ellipticity condition, then the problem (29) is well-posed; if additionally $\underline{\boldsymbol{\xi}} \in \underline{\mathbf{C}}^{1}(\bar{\Omega})$ and $\partial \Omega$ is of class $\mathcal{C}^{2}$, then, $\forall \sigma \in[0,1] \backslash\left\{\frac{1}{2}\right\}$,

$$
\ell \in\left(H_{0}^{1-\sigma}(\Omega)\right)^{\prime} \Longrightarrow p \in H^{\sigma+1}(\Omega)
$$

additionally,

$$
\exists C_{\sigma}>0, \forall \ell \in\left(H_{0}^{1-\sigma}(\Omega)\right)^{\prime},\|p\|_{\sigma+1, \Omega} \leq C_{\sigma}\|\ell\|_{\left(H_{0}^{1-\sigma}(\Omega)\right)^{\prime}}
$$

Consequently, we have the following regularity result for $\boldsymbol{E}$.

Theorem 3.4. Let $\boldsymbol{E}$, governed by (20), be split as $\boldsymbol{E}=\boldsymbol{E}_{0}+\boldsymbol{E}_{d}$ with $\boldsymbol{E}_{0} \in \boldsymbol{H}_{0}(\mathbf{c u r l} ; \Omega)$. If $\partial \Omega$ is of class $\mathcal{C}^{2}$, if $\underline{\boldsymbol{\varepsilon}} \in \underline{\mathbf{C}}^{1}(\bar{\Omega})$, if $\boldsymbol{f} \in \boldsymbol{L}^{2}(\Omega)$ is such that $\operatorname{div} \boldsymbol{f} \in H^{\mathbf{s}-1}(\Omega)$ with $\mathbf{s}$ in $[0,1] \backslash\left\{\frac{1}{2}\right\}$, and if $\boldsymbol{E}_{d} \in \boldsymbol{H}^{\mathrm{r}}(\Omega)$ with $\mathrm{r}$ in $[0,1] \backslash\left\{\frac{1}{2}\right\}$, then

$$
\begin{aligned}
& \boldsymbol{E} \in \boldsymbol{H}^{\min (\mathbf{s}, \mathbf{r})}(\Omega) \text { and } \\
& \|\boldsymbol{E}\|_{\min (\mathbf{s}, \mathbf{r}), \Omega} \lesssim\|\boldsymbol{f}\|_{0, \Omega}+\|\operatorname{div} \boldsymbol{f}\|_{\mathbf{s}-1, \Omega}+\left\|\boldsymbol{E}_{d}\right\|_{\mathrm{r}, \Omega}+\left\|\operatorname{curl} \boldsymbol{E}_{d}\right\|_{0, \Omega} .
\end{aligned}
$$

Remark 3.3. No regularity assumption on $\underline{\boldsymbol{\mu}}$ is required here.

Proof. We split $\boldsymbol{E}_{0}$ as in (27), and we apply theorem 3.3 to the problem (28) governing $\phi_{E}$. Let us introduce the right-hand side of $(28), \ell \in\left(H_{0}^{1}(\Omega)\right)^{\prime}$, and defined by

$$
\ell: q \mapsto\left(\operatorname{div} \boldsymbol{f}+\omega^{2} \operatorname{div} \underline{\boldsymbol{\varepsilon}} \boldsymbol{E}_{d}+\omega^{2} \operatorname{div} \underline{\boldsymbol{\varepsilon}} \boldsymbol{E}^{\mathrm{reg}} \mid q\right)_{0, \Omega} .
$$

Consider each term: one has $\operatorname{div} \boldsymbol{f} \in H^{\mathbf{s}-1}(\Omega)=\left(H_{0}^{1-\mathbf{s}}(\Omega)\right)^{\prime}$; as $\underline{\varepsilon} \in \underline{\mathbf{W}}^{1, \infty}(\Omega)$, there holds $\underline{\varepsilon} \boldsymbol{E}^{\mathrm{reg}} \in \boldsymbol{H}^{1}(\Omega)$ and $\operatorname{div} \underline{\boldsymbol{\varepsilon}} \boldsymbol{E}^{\mathrm{reg}} \in L^{2}(\Omega)$; similarly, $\underline{\boldsymbol{\varepsilon}} \boldsymbol{E}_{d} \in \boldsymbol{H}^{\mathrm{r}}(\Omega)$, so $\operatorname{div} \underline{\boldsymbol{\varepsilon}} \boldsymbol{E}_{d} \in H^{\mathrm{r}-1}(\Omega)=$ $\left(H_{0}^{1-\mathrm{r}}(\Omega)\right)^{\prime}$ as soon as $\mathrm{r} \neq \frac{1}{2}$. It follows that $\ell \in H^{\min (\mathbf{s}, \mathbf{r})-1}(\Omega)=\left(H_{0}^{1-\min (\mathbf{s}, \mathbf{r})}(\Omega)\right)^{\prime}$. In addition, one has the bound

$$
\begin{aligned}
\|\ell\|_{\min (\mathbf{s}, \mathbf{r})-1, \Omega} & \lesssim\|\operatorname{div} \boldsymbol{f}\|_{\mathbf{s}-1, \Omega}+\left\|\operatorname{div} \underline{\boldsymbol{\varepsilon}} \boldsymbol{E}^{\mathrm{reg}}\right\|_{0, \Omega}+\left\|\operatorname{div} \underline{\boldsymbol{\varepsilon}} \boldsymbol{E}_{d}\right\|_{\mathrm{r}-1, \Omega} \\
& \lesssim\|\operatorname{div} \boldsymbol{f}\|_{\mathbf{s}-1, \Omega}+\left\|\boldsymbol{E}^{\mathrm{reg}}\right\|_{1, \Omega}+\left\|\boldsymbol{E}_{d}\right\|_{\mathbf{r}, \Omega} \\
& \lesssim\|\operatorname{div} \boldsymbol{f}\|_{\mathbf{s}-1, \Omega}+\left\|\boldsymbol{E}_{0}\right\|_{\boldsymbol{H}(\mathbf{c u r l} ; \Omega)}+\left\|\boldsymbol{E}_{d}\right\|_{\mathbf{r}, \Omega},
\end{aligned}
$$

where we used (27) to reach the third line. We conclude by the shift theorem 3.3 that $\phi_{E} \in$ $H^{\min (\mathbf{s}, \mathbf{r})+1}(\Omega)$, and $\left\|\phi_{E}\right\|_{\min (\mathbf{s}, \mathbf{r})+1, \Omega} \lesssim\|\operatorname{div} \boldsymbol{f}\|_{\mathbf{s}-1, \Omega}+\left\|\boldsymbol{E}_{0}\right\|_{\boldsymbol{H}(\mathbf{c u r l} ; \Omega)}+\left\|\boldsymbol{E}_{d}\right\|_{\mathbf{r}, \Omega}$. Hence $\boldsymbol{E}=$ $\boldsymbol{E}^{\mathrm{reg}}+\nabla \phi_{E}+\boldsymbol{E}_{d}$ belongs to $\boldsymbol{H}^{\min (\mathbf{s}, \mathbf{r})}(\Omega)$, with the bound

$$
\begin{aligned}
\|\boldsymbol{E}\|_{\min (\mathrm{s}, \mathrm{r}), \Omega} & \lesssim\left\|\boldsymbol{E}^{\mathrm{reg}}\right\|_{1, \Omega}+\left\|\nabla \phi_{E}\right\|_{\min (\mathrm{s}, \mathrm{r}), \Omega}+\left\|\boldsymbol{E}_{d}\right\|_{\mathrm{r}, \Omega} \\
& \lesssim\left\|\boldsymbol{E}_{0}\right\|_{\boldsymbol{H}(\operatorname{curl} ; \Omega)}+\left\|\nabla \phi_{E}\right\|_{\min (\mathbf{s}, \mathbf{r}), \Omega}+\left\|\boldsymbol{E}_{d}\right\|_{\mathrm{r}, \Omega} \\
& \lesssim\left\|\boldsymbol{E}_{0}\right\|_{\boldsymbol{H}(\operatorname{curl} ; \Omega)}+\|\operatorname{div} \boldsymbol{f}\|_{\mathbf{s}-1, \Omega}+\left\|\boldsymbol{E}_{d}\right\|_{\mathrm{r}, \Omega}, \\
& \lesssim\|\boldsymbol{f}\|_{0, \Omega}+\left\|\operatorname{curl} \boldsymbol{E}_{d}\right\|_{0, \Omega}+\|\operatorname{div} \boldsymbol{f}\|_{\mathbf{s}-1, \Omega}+\left\|\boldsymbol{E}_{d}\right\|_{\mathrm{r}, \Omega},
\end{aligned}
$$

where we used successively (27), the bound on $\left\|\phi_{E}\right\|_{\min (\mathbf{s}, \mathbf{r})+1, \Omega}$ and finally (26) to conclude.

Corollary 3.1. Let the assumptions of theorem 3.4 hold. When $\boldsymbol{f} \in \boldsymbol{H}$ (div; $\Omega$ ), there holds $\boldsymbol{E} \in \boldsymbol{H}^{\mathrm{r}}(\Omega)$, and $\|\boldsymbol{E}\|_{\mathrm{r}, \Omega} \lesssim\|\boldsymbol{f}\|_{\boldsymbol{H}(\mathrm{div} ; \Omega)}+\left\|\boldsymbol{E}_{d}\right\|_{\mathrm{r}, \Omega}+\left\|\operatorname{curl} \boldsymbol{E}_{d}\right\|_{0, \Omega}$. 


\subsection{Extra-regularity of the solution's curl}

We proceed similarly with the solution's curl, with the same assumptions as in subsection 3.2. One has a theorem analogous to theorem 3.2, on the continuous splitting of fields of $\boldsymbol{H}(\mathbf{c u r l} ; \Omega)$, cf. Theorem 3.6.7 in Ref. [5] (see also Lemma 2.4 in Ref. [21] for a similar result):

Theorem 3.5. Let $\Omega$ be a domain of the $\mathfrak{A}$-type (see Definition A.2). For all $\boldsymbol{u}$ in $\boldsymbol{H}(\mathbf{c u r l} ; \Omega)$, there exist $\boldsymbol{u}^{\text {reg }}$ in $\boldsymbol{H}^{1}(\Omega)$ and $\phi$ in $H_{\mathrm{zmv}}^{1}(\Omega)$, such that $\boldsymbol{u}^{\text {reg }} \cdot \boldsymbol{n}_{\mid \partial \Omega}=0$ and

$$
\boldsymbol{u}=\boldsymbol{u}^{\mathrm{reg}}+\nabla \phi \text { in } \Omega, \text { with }\left\|\boldsymbol{u}^{\mathrm{reg}}\right\|_{1, \Omega}+\|\phi\|_{1, \Omega} \lesssim\|\boldsymbol{u}\|_{\boldsymbol{H}(\mathbf{c u r l} ; \Omega)}
$$

Let $\boldsymbol{C}:=\underline{\boldsymbol{\mu}}^{-1} \operatorname{curl} \boldsymbol{E} \in \boldsymbol{L}^{2}(\Omega)$, one has $\operatorname{curl} \boldsymbol{C}=\boldsymbol{f}+\omega^{2} \underline{\boldsymbol{\varepsilon}} \boldsymbol{E} \in \boldsymbol{L}^{2}(\Omega)$, hence $\boldsymbol{C} \in \boldsymbol{H}(\operatorname{curl} ; \Omega)$ with the bound

$$
\|\boldsymbol{C}\|_{\boldsymbol{H}(\operatorname{curl} ; \Omega)} \lesssim\|\boldsymbol{E}\|_{\boldsymbol{H}(\operatorname{curl} ; \Omega)}+\|\boldsymbol{f}\|_{0, \Omega},
$$

according to proposition 2.1. Observing that $\boldsymbol{E}=\boldsymbol{E}_{0}+\boldsymbol{E}_{d}$ with $\boldsymbol{E}_{0} \in \boldsymbol{H}_{0}(\mathbf{c u r l} ; \Omega)$, for any $q$ in $H_{\mathrm{zmv}}^{1}(\Omega)$, it holds that $(\underline{\boldsymbol{\mu}} \boldsymbol{C} \mid \nabla q)_{0, \Omega}=(\operatorname{curl} \boldsymbol{E} \mid \nabla q)_{0, \Omega}=\left(\operatorname{curl} \boldsymbol{E}_{d} \mid \nabla q\right)_{0, \Omega}$. Next, we introduce the splitting of $\boldsymbol{C}$ by theorem 3.5:

$$
\left\{\begin{array}{l}
\boldsymbol{C}=\boldsymbol{C}^{\mathrm{reg}}+\nabla \phi_{C}, \text { with } \boldsymbol{C}^{\mathrm{reg}} \in \boldsymbol{H}^{1}(\Omega), \phi_{C} \in H_{\mathrm{zmv}}^{1}(\Omega), \boldsymbol{C}^{\mathrm{reg}} \cdot \boldsymbol{n}_{\mid \partial \Omega}=0 \text { and } \\
\left\|\boldsymbol{C}^{\mathrm{reg}}\right\|_{1, \Omega}+\left\|\phi_{C}\right\|_{1, \Omega} \lesssim\|\boldsymbol{C}\|_{\boldsymbol{H}(\mathbf{c u r l} ; \Omega)} .
\end{array}\right.
$$

Thus $\phi_{C}$ is governed by the Neumann problem

$$
\left\{\begin{array}{l}
\text { Find } \phi_{C} \in H_{\mathrm{zmv}}^{1}(\Omega) \text { such that } \\
\left(\underline{\boldsymbol{\mu}} \nabla \phi_{C} \mid \nabla q\right)_{0, \Omega}=\left(\operatorname{curl} \boldsymbol{E}_{d}-\underline{\boldsymbol{\mu}} C^{\mathrm{reg}} \mid \nabla q\right)_{0, \Omega}, \forall q \in H_{\mathrm{zmv}}^{1}(\Omega) .
\end{array}\right.
$$

Hence, one may use a regularity result for solutions of the Neumann problem, see Theorem 3.4.5 in Ref. [14], to estimate the regularity of $\phi_{C}$, to infer the regularity of $\boldsymbol{C}$ and finally the regularity of $\operatorname{curl} \boldsymbol{E}$.

Theorem 3.6 (Shift theorem). Let $\Omega$ be a bounded domain of boundary $\partial \Omega, \ell$ in $\left(H_{\mathrm{zmv}}^{1}(\Omega)\right)^{\prime}$, and $p$ governed by

$$
\left\{\begin{array}{l}
\text { Find } p \in H_{\mathrm{zmv}}^{1}(\Omega) \text { such that } \\
(\underline{\boldsymbol{\xi}} \nabla p \mid \nabla q)_{0, \Omega}=\ell(q), \forall q \in H_{\mathrm{zmv}}^{1}(\Omega) .
\end{array}\right.
$$

If the tensor coefficient $\underline{\boldsymbol{\xi}}$ fulfills the ellipticity condition, then the problem (36) is well-posed; assume in addition that $\underline{\boldsymbol{\xi}} \in \underline{\mathbf{C}}^{1}(\bar{\Omega})$ and $\partial \Omega$ is of class $\mathcal{C}^{2}$.

(i) Then, $\forall \sigma \in\left[0, \frac{1}{2}[\right.$,

$$
\ell \in\left(H_{\mathrm{zmv}}^{1-\sigma}(\Omega)\right)^{\prime} \Longrightarrow p \in H^{\sigma+1}(\Omega)
$$

and,

$$
\exists C_{\sigma}>0, \forall \ell \in\left(H_{\mathrm{zmv}}^{1-\sigma}(\Omega)\right)^{\prime},\|p\|_{\sigma+1, \Omega} \leq C_{\sigma}\|\ell\|_{\left(H_{\mathrm{zmv}}^{1-\sigma}(\Omega)\right)^{\prime}} .
$$

(ii) If there exists $\left.\sigma \in] \frac{1}{2}, 1\right]$ such that $\ell$ writes $\ell(q)=(f \mid q)_{0, \Omega}+\langle g, q\rangle_{H^{1 / 2}(\partial \Omega)}$ with $f \in L^{2}(\Omega)$ and $g \in H^{\sigma-1 / 2}(\partial \Omega)$, then $p \in H^{\sigma+1}(\Omega)$. Moreover,

$$
\exists C_{\sigma}>0, \forall(f, g) \in L^{2}(\Omega) \times H^{\sigma-1 / 2}(\partial \Omega),\|p\|_{\sigma+1, \Omega} \leq C_{\sigma}\left(\|f\|_{0, \Omega}+\|g\|_{\sigma-1 / 2, \partial \Omega}\right) .
$$


Remark 3.4. In case (i), the theorem can be understood in a variational manner, just as in theorem 3.3 for the Dirichlet problem. On the other hand, in case (ii), the proof relies on local analysis arguments; see [14] for details.

Applying this result to problem (35), one finds the regularity of $\phi_{C}$, then of $\operatorname{curl} \boldsymbol{E}$.

Theorem 3.7. Let $\boldsymbol{E}$, governed by (20), be split as $\boldsymbol{E}=\boldsymbol{E}_{0}+\boldsymbol{E}_{d}$ with $\boldsymbol{E}_{0} \in \boldsymbol{H}_{0}(\mathbf{c u r l} ; \Omega)$. If $\partial \Omega$ is of class $\mathcal{C}^{2}$, if $\underline{\boldsymbol{\mu}} \in \underline{\mathbf{C}}^{1}(\bar{\Omega})$, and if $\operatorname{curl} \boldsymbol{E}_{d} \in \boldsymbol{H}^{\mathbf{r}^{\prime}}(\Omega)$ with $\mathbf{r}^{\prime}$ in $[0,1] \backslash\left\{\frac{1}{2}\right\}$, then

$$
\begin{aligned}
& \operatorname{curl} \boldsymbol{E} \in \boldsymbol{H}^{\mathrm{r}^{\prime}}(\Omega) \text { and } \\
& \|\operatorname{curl} \boldsymbol{E}\|_{\mathbf{r}^{\prime}, \Omega} \lesssim\|\boldsymbol{f}\|_{0, \Omega}+\left\|\boldsymbol{E}_{d}\right\|_{0, \Omega}+\left\|\operatorname{curl} \boldsymbol{E}_{d}\right\|_{\mathbf{r}^{\prime}, \Omega} .
\end{aligned}
$$

Remark 3.5. No regularity assumption on $\underline{\boldsymbol{\varepsilon}}$ (other than $\underline{\boldsymbol{\varepsilon}} \in \underline{\mathbf{L}}^{\infty}(\Omega)$ ) is required here. As $\Omega$ is a domain with boundary of class $\mathcal{C}^{2}$, it is automatically of the $\mathfrak{A}$-type.

Proof. We would like to apply theorem 3.6 to the problem (35) governing $\phi_{C}$. Let us introduce the right-hand side of $(35), \ell \in\left(H_{\mathrm{zmv}}^{1}(\Omega)\right)^{\prime}$, and defined by

$$
\ell: q \mapsto\left(\operatorname{curl} \boldsymbol{E}_{d}-\underline{\boldsymbol{\mu}} \boldsymbol{C}^{\mathrm{reg}} \mid \nabla q\right)_{0, \Omega} .
$$

To determine the regularity of $\phi_{C}$, one wants to determine whether the form $\ell$ belongs to $\left(H_{\mathrm{zmv}}^{1-\sigma}(\Omega)\right)^{\prime}$ for $\sigma \in[0,1] \backslash \frac{1}{2}$ as large as possible.

If $\boldsymbol{r}^{\prime}<\frac{1}{2}$, then $\boldsymbol{H}^{\mathbf{r}^{\prime}}(\Omega)$ identifies with $\boldsymbol{H}_{0}^{\mathbf{r}^{\prime}}(\Omega)$, the dual space of $\boldsymbol{H}^{-\mathbf{r}^{\prime}}(\Omega)$. Hence the product $\left(\operatorname{curl} \boldsymbol{E}_{d} \mid \nabla q\right)_{0, \Omega}$ is meaningful as soon as $q \in H_{\mathrm{zmv}}^{1-\mathrm{r}^{\prime}}(\Omega)$, because $\nabla q \in \boldsymbol{H}^{-\mathrm{r}^{\prime}}(\Omega)$ in this case; the same holds for the term $\left(\underline{\mu} C^{\text {reg }} \mid \nabla q\right)_{0, \Omega}$. This means that $\ell$ belongs to $\left(H_{\mathrm{zmv}}^{1-\mathbf{r}^{\prime}}(\Omega)\right)^{\prime}$, and the shift theorem 3.6 (i), with $\sigma=\mathrm{r}^{\prime}$, ensures that $\phi_{C} \in H^{1+\mathrm{r}^{\prime}}(\Omega)$, with the bound

$$
\left\|\phi_{C}\right\|_{1+\mathrm{r}^{\prime}, \Omega} \lesssim\left\|\operatorname{curl} \boldsymbol{E}_{d}\right\|_{\mathrm{r}^{\prime}, \Omega}+\left\|\underline{\boldsymbol{\mu}} \boldsymbol{C}^{\mathrm{reg}}\right\|_{1, \Omega} \lesssim\left\|\operatorname{curl} \boldsymbol{E}_{d}\right\|_{\mathrm{r}^{\prime}, \Omega}+\left\|\boldsymbol{C}^{\mathrm{reg}}\right\|_{1, \Omega} .
$$

On the other hand, if $\mathbf{r}^{\prime}>\frac{1}{2}$, then $\boldsymbol{H}_{0}^{\mathbf{r}^{\prime}}(\Omega)$ does not identify with $\boldsymbol{H}^{\mathrm{r}^{\prime}}(\Omega)$, and, as soon as $\operatorname{curl} \boldsymbol{E}_{d} \cdot \boldsymbol{n}_{\mid \partial \Omega} \neq 0$, the product $\left(\boldsymbol{c u r l} \boldsymbol{E}_{d} \mid \nabla q\right)_{0, \Omega}$ can be meaningless if one has only $q \in H_{\mathrm{zmv}}^{1-\mathrm{r}^{\prime}}(\Omega)$. However, since $\mathbf{r}^{\prime}>\frac{1}{2}$, curl $\boldsymbol{E}_{d} \cdot \boldsymbol{n}_{\mid \partial \Omega}$ makes sense in $H^{\mathrm{r}^{\prime}-1 / 2}(\partial \Omega)$, and, as $\underline{\boldsymbol{\mu}} \boldsymbol{C}^{\mathrm{reg}} \cdot \boldsymbol{n}_{\mid \partial \Omega}=0$, $\ell(q)$ rewrites by integrations by parts $\ell(q)=\left(\operatorname{div} \underline{\boldsymbol{\mu}} \boldsymbol{C}^{\mathrm{reg}} \mid q\right)_{0, \Omega}+\left\langle\operatorname{curl} \boldsymbol{E}_{d} \cdot \overline{\boldsymbol{n}}, q\right\rangle_{H^{1 / 2}(\partial \Omega)}$. As $\operatorname{div} \underline{\mu} C^{\text {reg }} \in L^{2}(\Omega), \ell$ satisfies the assumptions of the shift theorem 3.6 (ii) with $\sigma=\mathrm{r}^{\prime}$, and we conclude that $\phi_{C} \in H^{1+\mathrm{r}^{\prime}}(\Omega)$, with the bound

$$
\begin{aligned}
\left\|\phi_{C}\right\|_{1+\mathrm{r}^{\prime}, \Omega} & \lesssim\left\|\operatorname{curl} \boldsymbol{E}_{d} \cdot \boldsymbol{n}\right\|_{\mathrm{r}^{\prime}-1 / 2, \partial \Omega}+\left\|\operatorname{div} \underline{\boldsymbol{\mu}} \boldsymbol{C}^{\mathrm{reg}}\right\|_{0, \Omega} \\
& \lesssim\left\|\operatorname{curl} \boldsymbol{E}_{d}\right\|_{\mathrm{r}^{\prime}-1 / 2, \partial \Omega}+\left\|\operatorname{div} \underline{\boldsymbol{\mu}} \boldsymbol{C}^{\mathrm{reg}}\right\|_{0, \Omega} \\
& \lesssim\left\|\operatorname{curl} \boldsymbol{E}_{d}\right\|_{\mathrm{r}^{\prime}, \Omega}+\left\|\boldsymbol{C}^{\mathrm{reg}}\right\|_{1, \Omega} .
\end{aligned}
$$

Therefore, $\phi_{C} \in H^{1+\mathrm{r}^{\prime}}(\Omega)$ in all cases (with the same upper bound), so that $\boldsymbol{C}=\boldsymbol{C}^{\mathrm{reg}}+\nabla \phi_{C} \in$ $\boldsymbol{H}^{\mathrm{r}^{\prime}}(\Omega)$,

$$
\begin{aligned}
\|\boldsymbol{C}\|_{\mathrm{r}^{\prime}, \Omega} & \lesssim\left\|\boldsymbol{C}^{\mathrm{reg}}\right\|_{1, \Omega}+\left\|\nabla \phi_{C}\right\|_{\mathrm{r}^{\prime}, \Omega} \\
& \lesssim\left\|\boldsymbol{C}^{\mathrm{reg}}\right\|_{1, \Omega}+\left\|\operatorname{curl} \boldsymbol{E}_{d}\right\|_{\mathrm{r}^{\prime}, \Omega} \\
(\operatorname{see}(34)) & \lesssim\|\boldsymbol{C}\|_{\boldsymbol{H}(\operatorname{curl} ; \Omega)}+\left\|\operatorname{curl} \boldsymbol{E}_{d}\right\|_{\mathrm{r}^{\prime}, \Omega}
\end{aligned}
$$


As curl $\boldsymbol{E}=\boldsymbol{\mu} \boldsymbol{C}$ and $\boldsymbol{\mu} \in \underline{\mathbf{W}}^{1, \infty}(\Omega)$, it then holds that $\operatorname{curl} \boldsymbol{E} \in \boldsymbol{H}^{\mathrm{r}^{\prime}}(\Omega)$, with the bound $\|\operatorname{curl} \boldsymbol{E}\|_{\mathrm{r}^{\prime}, \Omega} \lesssim\|\boldsymbol{C}\|_{\mathrm{r}^{\prime}, \Omega}$. Finally, with the help of (33) and of theorem 3.1, one concludes that

$$
\begin{aligned}
\|\operatorname{curl} \boldsymbol{E}\|_{\mathrm{r}^{\prime}, \Omega} & \lesssim\|\boldsymbol{C}\|_{\boldsymbol{H}(\operatorname{curl} ; \Omega)}+\left\|\operatorname{curl} \boldsymbol{E}_{d}\right\|_{\mathrm{r}^{\prime}, \Omega} \\
& \lesssim\|\boldsymbol{E}\|_{\boldsymbol{H}(\operatorname{curl} ; \Omega)}+\|\boldsymbol{f}\|_{0, \Omega}+\left\|\operatorname{curl} \boldsymbol{E}_{d}\right\|_{\boldsymbol{r}^{\prime}, \Omega} \\
& \lesssim\left\|\boldsymbol{E}_{0}\right\|_{\boldsymbol{H}(\operatorname{curl} ; \Omega)}+\left\|\boldsymbol{E}_{d}\right\|_{0, \Omega}+\|\boldsymbol{f}\|_{0, \Omega}+\left\|\operatorname{curl} \boldsymbol{E}_{d}\right\|_{\mathbf{r}^{\prime}, \Omega} \\
& \lesssim\left\|\boldsymbol{E}_{d}\right\|_{0, \Omega}+\|\boldsymbol{f}\|_{0, \Omega}+\left\|\operatorname{curl} \boldsymbol{E}_{d}\right\|_{\mathrm{r}^{\prime}, \Omega} .
\end{aligned}
$$

The last theorem sums up the regularity results of this section.

Theorem 3.8. Let $\boldsymbol{E}$, governed by (20), be split as $\boldsymbol{E}=\boldsymbol{E}_{0}+\boldsymbol{E}_{d}$ with $\boldsymbol{E}_{0} \in \boldsymbol{H}_{0}(\mathbf{c u r l} ; \Omega)$. If $\partial \Omega$ is of class $\mathcal{C}^{2}$, if $\underline{\boldsymbol{\varepsilon}}, \boldsymbol{\mu} \in \underline{\mathbf{C}}^{1}(\bar{\Omega})$, if $\boldsymbol{f} \in \boldsymbol{L}^{2}(\Omega)$ and is such that $\operatorname{div} \boldsymbol{f} \in H^{\mathbf{s}-1}(\Omega)$ with $\mathbf{s}$ in $[0,1] \backslash\left\{\frac{1}{2}\right\}$, and if $\boldsymbol{E}_{d} \in \bar{H}^{\mathrm{r}}(\Omega)$ and is such that curl $\boldsymbol{E}_{d} \in \boldsymbol{H}^{\mathbf{r}^{\prime}}(\Omega)$ with $\mathbf{r}, \mathbf{r}^{\prime}$ in $[0,1] \backslash\left\{\frac{1}{2}\right\}$, then

$$
\left\{\begin{aligned}
\boldsymbol{E} \in \boldsymbol{H}^{\min (\mathbf{s}, \mathbf{r})}(\Omega), \operatorname{curl} \boldsymbol{E} \in \boldsymbol{H}^{\mathrm{r}^{\prime}}(\Omega), \text { and } \\
\|\boldsymbol{E}\|_{\min (\mathbf{s}, \mathbf{r}), \Omega} \lesssim\|\boldsymbol{f}\|_{0, \Omega}+\|\operatorname{div} \boldsymbol{f}\|_{\mathbf{s}-1, \Omega}+\left\|\boldsymbol{E}_{d}\right\|_{\mathrm{r}, \Omega}+\left\|\operatorname{curl} \boldsymbol{E}_{d}\right\|_{0, \Omega}, \\
\|\operatorname{curl} \boldsymbol{E}\|_{\mathrm{r}^{\prime}, \Omega} \lesssim\|\boldsymbol{f}\|_{0, \Omega}+\left\|\boldsymbol{E}_{d}\right\|_{0, \Omega}+\left\|\operatorname{curl} \boldsymbol{E}_{d}\right\|_{\mathbf{r}^{\prime}, \Omega} .
\end{aligned}\right.
$$

\section{Analysis of the Neumann problem}

In this section, the time-harmonic Maxwell's equation is supplemented by a Neumann boundary condition:

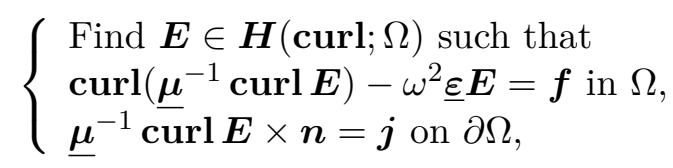

where $\boldsymbol{j}$ is a boundary data which can be interpreted as a surface current.

We assume that the tensors $\underline{\varepsilon}, \boldsymbol{\mu} \in \underline{\mathbf{L}}^{\infty}(\Omega)$ are elliptic. The corresponding coercivity directions (or, equivalently, the parameters $\theta_{\epsilon}$ and $\theta_{\mu}$ in definition 2.1) may be different. We also assume that $\boldsymbol{f} \in \boldsymbol{L}^{2}(\Omega)$ and that $\boldsymbol{j}$ is the tangential trace of a field $\boldsymbol{B}_{d}$ defined on $\Omega$, i.e. $\boldsymbol{j}=\boldsymbol{B}_{d} \times \boldsymbol{n}_{\mid \partial \Omega}$, with $\boldsymbol{B}_{d} \in \boldsymbol{H}(\operatorname{curl}, \Omega)$.

\subsection{Variational formulation and well-posedness}

The equivalent variational formulation of the problem is obtained by the integration by parts formula (1). It writes:

$$
\left\{\begin{array}{l}
\text { Find } \boldsymbol{E} \in \boldsymbol{H}(\operatorname{curl} ; \Omega) \text { such that } \\
\left(\underline{\boldsymbol{\mu}}^{-1} \operatorname{curl} \boldsymbol{E} \mid \operatorname{curl} \boldsymbol{F}\right)_{0, \Omega}-\omega^{2}(\underline{\boldsymbol{\varepsilon}} \boldsymbol{E} \mid \boldsymbol{F})_{0, \Omega}=\ell_{\mathrm{N}}(\boldsymbol{F}), \forall \boldsymbol{F} \in \boldsymbol{H}(\operatorname{curl} ; \Omega),
\end{array}\right.
$$

where $\ell_{\mathrm{N}}: \boldsymbol{F} \mapsto(\boldsymbol{f} \mid \boldsymbol{F})_{0, \Omega}-{ }_{\gamma}\left\langle\boldsymbol{j}, \pi^{T} \boldsymbol{F}\right\rangle_{\pi}$ belongs to $(\boldsymbol{H}(\mathbf{c u r l} ; \Omega))^{\prime}$, and

$$
\left\|\ell_{\mathrm{N}}\right\|_{(\boldsymbol{H}(\operatorname{curl} ; \Omega))^{\prime}} \lesssim\|\boldsymbol{f}\|_{0, \Omega}+\|\boldsymbol{j}\|_{\gamma} \lesssim\|\boldsymbol{f}\|_{0, \Omega}+\left\|\boldsymbol{B}_{d}\right\|_{\boldsymbol{H}(\mathbf{c u r l} ; \Omega)} .
$$

The analysis follows the same reasoning as in the section 3. For this reason, some proofs are just outlined. For the Neumann problem, our analysis relies on a second-kind Helmholtz decomposition (see theorem 2.3). 
Lemma 4.1. The formulation (43) can be equivalently recast as follows: set $\boldsymbol{E}=\tilde{\boldsymbol{E}}+\nabla p$, with $p \in H_{\mathrm{zmv}}^{1}(\Omega)$ and $\tilde{\boldsymbol{E}} \in \boldsymbol{K}_{T}(\underline{\varepsilon} ; \Omega)$, respectively governed by

$$
\left\{\begin{array}{l}
\text { Find } p \in H_{\mathrm{zmv}}^{1}(\Omega) \text { such that } \\
-\omega^{2}(\underline{\varepsilon} \nabla p \mid \nabla q)_{0, \Omega}=\ell_{\mathrm{N}}(\nabla q), \forall q \in H_{\mathrm{zmv}}^{1}(\Omega)
\end{array}\right.
$$

and

$$
\left\{\begin{array}{l}
\text { Find } \tilde{\boldsymbol{E}} \in \boldsymbol{K}_{T}(\underline{\varepsilon} ; \Omega) \text { such that } \\
\left(\underline{\boldsymbol{\mu}}^{-1} \operatorname{curl} \tilde{\boldsymbol{E}} \mid \operatorname{curl} \tilde{\boldsymbol{F}}\right)_{0, \Omega}-\omega^{2}(\underline{\varepsilon} \tilde{\boldsymbol{E}} \mid \tilde{\boldsymbol{F}})_{0, \Omega}=\omega^{2}(\underline{\varepsilon} \nabla p \mid \tilde{\boldsymbol{F}})_{0, \Omega}+\ell_{\mathrm{N}}(\tilde{\boldsymbol{F}}), \forall \tilde{\boldsymbol{F}} \in \boldsymbol{K}_{T}(\underline{\varepsilon} ; \Omega) .
\end{array}\right.
$$

Proof. Direct. Let us introduce the second-kind Helmholtz decomposition (9) of $\boldsymbol{E}: \boldsymbol{E}=$ $\tilde{\boldsymbol{E}}+\nabla p$, with $p \in H_{\mathrm{zmv}}^{1}(\Omega)$ and $\tilde{\boldsymbol{E}} \in \boldsymbol{K}_{T}(\underline{\boldsymbol{\varepsilon}} ; \Omega)$. Taking $\boldsymbol{F}=\nabla q$ in $(43)$ for any $q \in H_{\mathrm{zmv}}^{1}(\Omega)$ yields

$$
-\omega^{2}(\underline{\varepsilon}(\tilde{\boldsymbol{E}}+\nabla p) \mid \nabla q)_{0, \Omega}=\ell_{\mathrm{N}}(\nabla q) .
$$

Hence, as $\tilde{\boldsymbol{E}}$ belongs to $\boldsymbol{H}_{0}(\operatorname{div} \underline{\varepsilon} 0 ; \Omega), p$ is governed by $(45)$.

On the other hand, there holds

$$
\left(\underline{\boldsymbol{\mu}}^{-1} \operatorname{curl} \tilde{\boldsymbol{E}} \mid \operatorname{curl} \boldsymbol{F}\right)_{0, \Omega}-\omega^{2}(\underline{\varepsilon} \tilde{\boldsymbol{E}} \mid \boldsymbol{F})_{0, \Omega}=\omega^{2}(\underline{\varepsilon} \nabla p \mid \boldsymbol{F})_{0, \Omega}+\ell_{\mathrm{N}}(\boldsymbol{F})
$$

for any $\boldsymbol{F} \in \boldsymbol{H}(\operatorname{curl} ; \Omega)$, hence for any $\tilde{\boldsymbol{F}} \in \boldsymbol{K}_{T}(\underline{\varepsilon} ; \Omega)$ : $\tilde{\boldsymbol{E}}$ is governed by (46).

Reverse. Summing (45) and (46) and introducing $\boldsymbol{E}:=\tilde{\boldsymbol{E}}+\nabla p \in \boldsymbol{H}(\mathbf{c u r l} ; \Omega)$, one gets, after rearrangements:

$$
\left(\underline{\boldsymbol{\mu}}^{-1} \operatorname{curl} \boldsymbol{E} \mid \operatorname{curl}(\tilde{\boldsymbol{F}}+\nabla q)\right)_{0, \Omega}-\omega^{2}(\underline{\varepsilon} \boldsymbol{E} \mid(\tilde{\boldsymbol{F}}+\nabla q))_{0, \Omega}=\ell_{\mathrm{N}}(\tilde{\boldsymbol{F}}+\nabla q) .
$$

As $q$ and $\tilde{\boldsymbol{F}}$ span respectively $H_{\mathrm{zmv}}^{1}(\Omega)$ and $\boldsymbol{K}_{T}(\underline{\boldsymbol{\varepsilon}} ; \Omega)$, we know that the sum $\tilde{\boldsymbol{F}}+\nabla q$ spans the whole $\boldsymbol{H}(\mathbf{c u r l} ; \Omega)$ thanks to (9); hence $\boldsymbol{E}$ is governed by (43).

Here, $\boldsymbol{K}_{T}(\underline{\varepsilon} ; \Omega)$ is equipped with the norm $\|\cdot\|_{\boldsymbol{H}(\mathbf{c u r l} ; \Omega)}$. The rest of the analysis proceeds as for the Dirichlet problem.

Theorem 4.1. The formulation (43) enters Fredholm's alternative:

- either the problem (43) admits a unique solution $\boldsymbol{E}$ in $\boldsymbol{H}(\mathbf{c u r l} ; \Omega)$, which depends continuously on the data $\boldsymbol{f}$ and $\boldsymbol{B}_{d}$ :

$$
\|\boldsymbol{E}\|_{\boldsymbol{H}(\operatorname{curl} ; \Omega)} \lesssim\|\boldsymbol{f}\|_{0, \Omega}+\left\|\boldsymbol{B}_{d}\right\|_{\boldsymbol{H}(\operatorname{curl} ; \Omega)}
$$

- or, the problem (43) has solutions if, and only if, $\boldsymbol{f}$ and $\boldsymbol{B}_{d}$ satisfy a finite number of compatibility conditions; in this case, the space of solutions is an affine space of finite dimension. Additionally, the component of the solution which is orthogonal (in the sense of the $\boldsymbol{H}(\mathbf{c u r l} ; \Omega)$ scalar product) to the corresponding linear vector space, depends continuously on the data $\boldsymbol{f}$ and $\boldsymbol{B}_{d}$. 
Proof. The Neumann problem (45) is well-posed according to theorem 2.1. In fact, the form $\ell_{\mathrm{N}}$ is continuous on $\boldsymbol{H}(\mathbf{c u r l} ; \Omega)$ and the mapping $\nabla$ is continuous from $H_{\mathrm{zmv}}^{1}(\Omega)$ to $\boldsymbol{H}(\operatorname{curl} ; \Omega)$, so one has $\ell_{\mathrm{N}} \circ \nabla \in\left(H_{\mathrm{zmv}}^{1}(\Omega)\right)^{\prime}$, with the bound $\|p\|_{1, \Omega} \lesssim\|\boldsymbol{f}\|_{0, \Omega}+\left\|\boldsymbol{B}_{d}\right\|_{\boldsymbol{H}(\mathbf{c u r l} ; \Omega)}$.

In addition, the formulation (46) fits the coercive + compact framework. Indeed, as in the proof of lemma 3.3, one can split the left-hand side of (46) in two terms. Let $\alpha>0$, the term $\left(\underline{\boldsymbol{\mu}}^{-1} \operatorname{curl} \tilde{\boldsymbol{E}} \mid \operatorname{curl} \tilde{\boldsymbol{F}}\right)_{0, \Omega}+\alpha e^{i \theta_{\mu}}(\tilde{\boldsymbol{E}} \mid \tilde{\boldsymbol{F}})_{0, \Omega}$ is coercive on $\boldsymbol{H}(\operatorname{curl} ; \Omega)$ (therefore on $\boldsymbol{K}_{T}(\underline{\varepsilon} ; \Omega)$ ), as $\underline{\boldsymbol{\mu}}^{-1}$ satisfies the ellipticity condition (see proposition 2.1). The remaining term $-\omega^{2}(\underline{\varepsilon} \tilde{\boldsymbol{E}} \mid \tilde{\boldsymbol{F}})_{0, \Omega}-\alpha e^{i \theta_{\mu}}(\tilde{\boldsymbol{E}} \mid \tilde{\boldsymbol{F}})_{0, \Omega}$ is continuous on $\boldsymbol{L}^{2}(\Omega) \times \boldsymbol{K}_{T}(\underline{\varepsilon} ; \Omega)$, and the embedding of $\boldsymbol{K}_{T}(\underline{\varepsilon} ; \Omega)$ into $\boldsymbol{L}^{2}(\Omega)$ is compact by theorem 2.7. Hence formulation (46) enters the coercive + compact framework, and so does formulation (43). The bound $\|\tilde{\boldsymbol{E}}\|_{\boldsymbol{H}(\mathbf{c u r l} ; \Omega)} \lesssim\|\nabla p\|_{0, \Omega}+$ $\|\boldsymbol{f}\|_{0, \Omega}+\left\|\boldsymbol{B}_{d}\right\|_{\boldsymbol{H}(\mathbf{c u r l} ; \Omega)}$ follows from (44), and the bound on $\|\boldsymbol{E}\|_{\boldsymbol{H}(\mathbf{c u r l} ; \Omega)}$ is a consequence of the triangle inequality.

Assumption. In the rest of the manuscript, we assume that the problem (43) is well-posed and, in particular, (47) holds.

\subsection{Extra-regularity of the solution's curl}

As previously, we are now willing to estimate the regularity of the solution and its curl in the Neumann case, depending on the extra-regularity of the data. We make the following extraregularity assumptions for the next two subsections. We assume that $\partial \Omega$ is of class $\mathcal{C}^{2}$, and that $\underline{\boldsymbol{\varepsilon}} \in \underline{\mathbf{W}}^{1, \infty}(\Omega)$ and $\underline{\boldsymbol{\mu}} \in \underline{\mathbf{C}}^{1}(\bar{\Omega})$. Regarding the extra-regularity of $\boldsymbol{f}$ and $\boldsymbol{B}_{d}$, we assume that $\boldsymbol{f} \in \boldsymbol{H}(\mathbf{c u r l} ; \Omega) \cap \overline{\boldsymbol{H}}^{\mathrm{s}}(\Omega)$ for a given $\mathrm{s} \in[0,1]$, and that $\boldsymbol{B}_{d} \in \boldsymbol{H}^{\mathrm{r}^{\prime}}(\Omega)$ and $\operatorname{curl} \boldsymbol{B}_{d} \in \boldsymbol{H}^{\mathrm{r}}(\Omega)$ for given $r^{\prime}, r \in[0,1] \backslash\left\{\frac{1}{2}\right\}$. We begin with the estimation on the curl. The analysis relies on the same arguments as in subsection 3.2.

Let us introduce $\boldsymbol{B}:=\underline{\boldsymbol{\mu}}^{-1} \operatorname{curl} \boldsymbol{E} \in \boldsymbol{L}^{2}(\Omega)$. There holds $\operatorname{curl} \boldsymbol{B}=\boldsymbol{f}+\omega^{2} \underline{\boldsymbol{\varepsilon}} \boldsymbol{E} \in \boldsymbol{L}^{2}(\Omega)$, hence $\boldsymbol{B} \in \boldsymbol{H}(\operatorname{curl} ; \Omega)$, with the bound $\|\boldsymbol{B}\|_{\boldsymbol{H}(\operatorname{curl} ; \Omega)} \lesssim\|\boldsymbol{E}\|_{\boldsymbol{H}(\mathbf{c u r l} ; \Omega)}+\|\boldsymbol{f}\|_{0, \Omega}$, according to proposition 2.1. Moreover, $\boldsymbol{B} \times \boldsymbol{n}=\boldsymbol{B}_{d} \times \boldsymbol{n}$ on $\partial \Omega$. Letting $\boldsymbol{B}_{0}:=\boldsymbol{B}-\boldsymbol{B}_{d} \in \boldsymbol{H}_{0}(\mathbf{c u r l} ; \Omega)$, we introduce the splitting of $\boldsymbol{B}_{0}$ by theorem 3.2:

$$
\left\{\begin{array}{l}
\boldsymbol{B}_{0}=\boldsymbol{B}^{\text {reg }}+\nabla \phi_{B}, \text { with } \boldsymbol{B}^{\text {reg }} \in \boldsymbol{H}^{1}(\Omega), \phi_{B} \in H_{0}^{1}(\Omega), \text { and } \\
\left\|\boldsymbol{B}^{\text {reg }}\right\|_{1, \Omega}+\left\|\phi_{B}\right\|_{1, \Omega} \lesssim\left\|\boldsymbol{B}_{0}\right\|_{\boldsymbol{H}(\mathbf{c u r l} ; \Omega)} .
\end{array}\right.
$$

As $\operatorname{div} \boldsymbol{\mu} \boldsymbol{B}=0, \phi_{B}$ is governed by the Dirichlet problem

$$
\left\{\begin{array}{l}
\text { Find } \phi_{B} \in H_{0}^{1}(\Omega) \text { such that } \\
\left(\underline{\boldsymbol{\mu}} \nabla \phi_{B} \mid \nabla q\right)_{0, \Omega}=\left(\operatorname{div} \underline{\boldsymbol{\mu}} \boldsymbol{B}_{d}+\operatorname{div} \underline{\boldsymbol{\mu}}^{\mathrm{reg}} \mid q\right)_{0, \Omega}, \forall q \in H_{0}^{1}(\Omega) .
\end{array}\right.
$$

As in subsection 3.2 , one can apply the shift theorem 3.3 to get the regularity estimate on $\phi_{B}$ and $\operatorname{curl} \boldsymbol{E}$.

Theorem 4.2. Let $\boldsymbol{E}$ be governed by (42), and $\boldsymbol{B}=\boldsymbol{\mu}^{-1}$ curl $\boldsymbol{E}$ be split as $\boldsymbol{B}=\boldsymbol{B}_{0}+\boldsymbol{B}_{d}$ with $\boldsymbol{B}_{0} \in \boldsymbol{H}_{0}(\mathbf{c u r l} ; \Omega)$. If $\partial \Omega$ is of class $\mathcal{C}^{2}$, if $\underline{\boldsymbol{\mu}} \in \underline{\mathbf{C}}^{1}(\bar{\Omega})$, and if $\boldsymbol{B}_{d} \in \boldsymbol{H}^{\mathbf{r}^{\prime}}(\Omega)$ with $\boldsymbol{r}^{\prime} \in[0,1] \backslash\left\{\frac{1}{2}\right\}$, then

$$
\begin{aligned}
& \operatorname{curl} \boldsymbol{E} \in \boldsymbol{H}^{\mathrm{r}^{\prime}}(\Omega) \text { and } \\
& \|\operatorname{curl} \boldsymbol{E}\|_{\mathbf{r}^{\prime}, \Omega} \lesssim\|\boldsymbol{f}\|_{0, \Omega}+\left\|\operatorname{curl} \boldsymbol{B}_{d}\right\|_{0, \Omega}+\left\|\boldsymbol{B}_{d}\right\|_{\mathbf{r}^{\prime}, \Omega} .
\end{aligned}
$$


Remark 4.1. No regularity assumption on $\underline{\boldsymbol{\varepsilon}}$ (other than $\underline{\boldsymbol{\varepsilon}} \in \underline{\mathbf{L}}^{\infty}(\Omega)$ ) is required here.

Proof. The proof proceeds as in theorem 3.4. We apply the shift theorem 3.3 to the Dirichlet problem (49) governing $\phi_{B}$. Let us introduce the right-hand side of $(49), \ell \in\left(H_{0}^{1}(\Omega)\right)^{\prime}$, and defined by

$$
\ell: q \mapsto\left(\operatorname{div} \underline{\boldsymbol{\mu}} \boldsymbol{B}_{d}+\operatorname{div} \underline{\boldsymbol{\mu}} \boldsymbol{B}^{\mathrm{reg}} \mid q\right)_{0, \Omega} .
$$

We observe that there holds, as $\underline{\boldsymbol{\mu}} \in \underline{\mathbf{W}}^{1, \infty}(\Omega)$, that $\operatorname{div} \underline{\boldsymbol{\mu}} \boldsymbol{B}^{\mathrm{reg}} \in L^{2}(\Omega)$ and $\operatorname{div} \underline{\boldsymbol{\mu}} \boldsymbol{B}_{d} \in H^{\mathrm{r}^{\prime}-1}(\Omega)$ (as soon as $\mathrm{r}^{\prime} \neq \frac{1}{2}$ for the latter). Then $\ell \in H^{\mathrm{r}^{\prime}-1}(\Omega)=\left(H_{0}^{1-\mathrm{r}^{\prime}}(\Omega)\right)^{\prime}$. Hence, by the shift theorem 3.3, we have that $\phi_{B} \in H^{1+r^{\prime}}(\Omega)$. Moreover, thanks to (47), we have the bound

$$
\left\|\phi_{B}\right\|_{1+\mathrm{r}^{\prime}, \Omega} \lesssim\|\boldsymbol{f}\|_{0, \Omega}+\left\|\operatorname{curl} \boldsymbol{B}_{d}\right\|_{0, \Omega}+\left\|\boldsymbol{B}_{d}\right\|_{\mathrm{r}^{\prime}, \Omega} .
$$

So, $\boldsymbol{B}=\boldsymbol{B}^{\text {reg }}+\nabla \phi_{B}+\boldsymbol{B}_{d} \in \boldsymbol{H}^{\mathrm{r}^{\prime}}(\Omega)$ and we conclude that (50) holds, because $\underline{\boldsymbol{\mu}} \in \underline{\mathbf{W}}^{1, \infty}(\Omega)$.

\subsection{Extra-regularity of the solution}

To estimate the regularity of the solution itself, we follow the same approach as in subsection 3.3, with the same assumptions as in subsection 4.2. To that end, let us introduce $\boldsymbol{G}:=\operatorname{curl} \boldsymbol{B}=$ $\boldsymbol{f}+\omega^{2} \underline{\varepsilon} \boldsymbol{E} \in \boldsymbol{L}^{2}(\Omega)$. As $\underline{\varepsilon} \in \underline{\mathbf{W}}^{1, \infty}(\Omega)$ and $\boldsymbol{f} \in \boldsymbol{H}(\operatorname{curl} ; \Omega)$, one finds that $\operatorname{curl} \boldsymbol{G}=\operatorname{curl} \boldsymbol{f}+$ $\omega^{2} \operatorname{curl} \underline{\varepsilon} \boldsymbol{E} \in \boldsymbol{L}^{2}(\Omega)$, hence $\boldsymbol{G} \in \boldsymbol{H}(\operatorname{curl} ; \Omega)$, with the bound $\|\boldsymbol{G}\|_{\boldsymbol{H}(\operatorname{curl} ; \Omega)} \lesssim\|\boldsymbol{E}\|_{\boldsymbol{H}(\operatorname{curl} ; \Omega)}+$ $\|\boldsymbol{f}\|_{\boldsymbol{H}(\mathbf{c u r l} ; \Omega)}$. By theorem 3.5, one can introduce the splitting of $\boldsymbol{G}$ :

$$
\left\{\begin{array}{l}
\boldsymbol{G}=\boldsymbol{G}^{\mathrm{reg}}+\nabla \phi_{G}, \text { with } \boldsymbol{G}^{\mathrm{reg}} \in \boldsymbol{H}^{1}(\Omega), \phi_{G} \in H_{\mathrm{zmv}}^{1}(\Omega), \boldsymbol{G}^{\mathrm{reg}} \cdot \boldsymbol{n}_{\mid \partial \Omega}=0 \text { and } \\
\left\|\boldsymbol{G}^{\mathrm{reg}}\right\|_{1, \Omega}+\left\|\phi_{G}\right\|_{1, \Omega} \lesssim\|\boldsymbol{G}\|_{\boldsymbol{H}(\mathbf{c u r l} ; \Omega)} .
\end{array}\right.
$$

Therefore, for any $q \in H_{\mathrm{zmv}}^{1}(\Omega)$, there holds $(\boldsymbol{G} \mid \nabla q)_{0, \Omega}=(\operatorname{curl} \boldsymbol{B} \mid \nabla q)_{0, \Omega}=\left(\operatorname{curl} \boldsymbol{B}_{d} \mid \nabla q\right)_{0, \Omega}$, as the part with $\boldsymbol{B}_{0} \in \boldsymbol{H}_{0}(\mathbf{c u r l} ; \Omega)$ vanishes by integration by parts. Then $\phi_{G}$ is governed by the Neumann problem

$$
\left\{\begin{array}{l}
\text { Find } \phi_{G} \in H_{\mathrm{zmv}}^{1}(\Omega) \text { such that } \\
\left(\nabla \phi_{G} \mid \nabla q\right)_{0, \Omega}=\left(\operatorname{curl} \boldsymbol{B}_{d}-\boldsymbol{G}^{\mathrm{reg}} \mid \nabla q\right)_{0, \Omega}, \forall q \in H_{\mathrm{zmv}}^{1}(\Omega) .
\end{array}\right.
$$

As in subsection 3.3, the regularity of $\phi_{G}$, and $\boldsymbol{E}$, is then given by the shift theorem 3.6.

Theorem 4.3. Let $\boldsymbol{E}$ be governed by (42), and $\boldsymbol{B}=\boldsymbol{\mu}^{-1}$ curl $\boldsymbol{E}$ be split as $\boldsymbol{B}=\boldsymbol{B}_{0}+\boldsymbol{B}_{d}$ with $\boldsymbol{B}_{0} \in \boldsymbol{H}_{0}(\mathbf{c u r l} ; \Omega)$. If $\partial \Omega$ is of class $\mathcal{C}^{2}$, if $\underline{\varepsilon} \in \underline{\mathbf{W}}^{1, \infty}(\Omega)$, if $\boldsymbol{f} \in \boldsymbol{H}(\operatorname{curl} ; \Omega) \cap \boldsymbol{H}^{\mathrm{s}}(\Omega)$ with $\mathbf{s} \in[0,1]$, and if $\operatorname{curl} \boldsymbol{B}_{d} \in \boldsymbol{H}^{\mathrm{r}}(\Omega)$ with $\mathrm{r} \in[0,1] \backslash\left\{\frac{1}{2}\right\}$, then

$$
\begin{aligned}
& \boldsymbol{E} \in \boldsymbol{H}^{\min (\mathrm{r}, \mathbf{s})}(\Omega) \text { and } \\
& \|\boldsymbol{E}\|_{\min (\mathrm{r}, \mathbf{s}), \Omega} \lesssim\|\operatorname{curl} \boldsymbol{f}\|_{0, \Omega}+\|\boldsymbol{f}\|_{\mathbf{s}, \Omega}+\left\|\boldsymbol{B}_{d}\right\|_{0, \Omega}+\left\|\operatorname{curl} \boldsymbol{B}_{d}\right\|_{\mathrm{r}, \Omega} .
\end{aligned}
$$

Remark 4.2. No regularity assumption on $\underline{\boldsymbol{\mu}}$ is required here.

Proof. The proof is as in theorem 3.7: we want to apply the shift theorem 3.6 to the problem (52) governing $\phi_{G}$. Let us introduce the right-hand side of $(52), \ell \in\left(H_{\mathrm{zmv}}^{1}(\Omega)\right)^{\prime}$, and defined by

$$
\ell: q \mapsto\left(\operatorname{curl} \boldsymbol{B}_{d}-\boldsymbol{G}^{\mathrm{reg}} \mid \nabla q\right)_{0, \Omega}
$$

To determine the regularity of $\phi_{G}$, one wants to determine whether the form $\ell$ belongs to $\left(H_{\mathrm{zmv}}^{1-\sigma}(\Omega)\right)^{\prime}$ for $\sigma \in[0,1] \backslash \frac{1}{2}$ as large as possible. 
If $\mathbf{r}<\frac{1}{2}$, then $\boldsymbol{H}^{\mathrm{r}}(\Omega)$ identifies with $\boldsymbol{H}_{0}^{\mathrm{r}}(\Omega)$, the dual space of $\boldsymbol{H}^{-\mathrm{r}}(\Omega)$. Hence the product (curl $\left.\boldsymbol{B}_{d}-\boldsymbol{G}^{\mathrm{reg}} \mid \nabla q\right)$ is meaningful as soon as $q \in H_{\mathrm{zmv}}^{1-\mathrm{r}}(\Omega)$, because $\nabla q \in \boldsymbol{H}^{-\mathrm{r}}(\Omega)$ in this case. This means that $\ell$ belongs to $\left(H_{\mathrm{zmv}}^{1-\mathrm{r}}(\Omega)\right)^{\prime}$, and the shift theorem 3.6 (i), with $\sigma=\mathrm{r}$, ensures that $\phi_{G} \in H^{1+\mathrm{r}}(\Omega)$, with the bound

$$
\left\|\phi_{G}\right\|_{1+\mathrm{r}, \Omega} \lesssim\|\boldsymbol{f}\|_{\boldsymbol{H}(\operatorname{curl} ; \Omega)}+\left\|\operatorname{curl} \boldsymbol{B}_{d}\right\|_{\mathrm{r}, \Omega}+\left\|\boldsymbol{B}_{d}\right\|_{0, \Omega} .
$$

On the other hand, if $\mathbf{r}>\frac{1}{2}$, the previous argument is not valid anymore. However, $\operatorname{curl} \boldsymbol{B}_{d} \cdot \boldsymbol{n}_{\mid \partial \Omega}$ makes sense in $H^{\mathrm{r}-1 / 2}(\partial \Omega)$, and, as $\boldsymbol{G}^{\mathrm{reg}} \cdot \boldsymbol{n}_{\mid \partial \Omega}=0, \ell(q)$ rewrites by integrations by parts $\ell(q)=\left(\operatorname{div} \boldsymbol{G}^{\mathrm{reg}} \mid q\right)_{0, \Omega}+\left\langle\operatorname{curl} \boldsymbol{B}_{d} \cdot \boldsymbol{n}, q\right\rangle_{H^{1 / 2}(\partial \Omega)}$. As $\operatorname{div} \boldsymbol{G}^{\mathrm{reg}} \in L^{2}(\Omega), \ell$ satisfies the assumptions of the shift theorem 3.6 (ii), with $\sigma=\mathrm{r}$, and we conclude that $\phi_{G} \in H^{1+\mathrm{r}}(\Omega)$, with the same bound as above.

Finally, $\phi_{G} \in H^{1+\mathrm{r}}(\Omega)$ in all cases, and $\boldsymbol{G}=\boldsymbol{G}^{\mathrm{reg}}+\nabla \phi_{G} \in \boldsymbol{H}^{\mathrm{r}}(\Omega)$. In passing, we note that $\underline{\varepsilon}^{-1} \in \underline{\mathbf{W}}^{1, \infty}(\Omega)$, because $\underline{\varepsilon}^{-1} \in \underline{\mathbf{L}}^{\infty}(\Omega)$ (Proposition 2.1) and $\underline{\varepsilon} \in \underline{\mathbf{W}}^{1, \infty}(\Omega)$. Recalling that $\boldsymbol{E}=\omega^{-2} \underline{\varepsilon}^{-1}(\boldsymbol{G}-\boldsymbol{f})$, with $\boldsymbol{f} \in \boldsymbol{H}^{\mathrm{s}}(\Omega)$, we conclude that (53) holds.

To conclude, we sum up the regularity results of this section.

Theorem 4.4. Let $\boldsymbol{E}$ be governed by problem (42), and $\boldsymbol{B}=\underline{\boldsymbol{\mu}}^{-1} \operatorname{curl} \boldsymbol{E}$ be split as $\boldsymbol{B}=$ $\boldsymbol{B}_{0}+\boldsymbol{B}_{d}$ with $\boldsymbol{B}_{0} \in \boldsymbol{H}_{0}(\mathbf{c u r l} ; \Omega)$. If $\partial \Omega$ is of class $\mathcal{C}^{2}$, if $\underline{\boldsymbol{\mu}} \in \underline{\mathbf{C}}^{1}(\bar{\Omega})$, if $\underline{\boldsymbol{\varepsilon}} \in \underline{\mathbf{W}}^{1, \infty}(\Omega)$, if $\boldsymbol{f} \in \boldsymbol{H}(\mathbf{c u r l} ; \Omega) \cap \boldsymbol{H}^{\mathrm{s}}(\Omega)$ with $\mathbf{s}$ in $[0,1]$, and if $\boldsymbol{B}_{d} \in \boldsymbol{H}^{\mathrm{r}^{\prime}}(\Omega)$ and is such that $\mathbf{c u r l} \boldsymbol{B}_{d} \in \boldsymbol{H}^{\mathrm{r}}(\Omega)$ with $\mathbf{r}^{\prime}, \mathbf{r}$ in $[0,1] \backslash \frac{1}{2}$, then

$$
\left\{\begin{array}{l}
\boldsymbol{E} \in \boldsymbol{H}^{\min (\mathrm{r}, \mathbf{s})}(\Omega), \quad \operatorname{curl} \boldsymbol{E} \in \boldsymbol{H}^{\mathrm{r}^{\prime}}(\Omega), \text { and } \\
\|\boldsymbol{E}\|_{\min (\mathrm{r}, \mathrm{s}), \Omega} \lesssim\|\boldsymbol{f}\|_{\mathrm{s}, \Omega}+\|\operatorname{curl} \boldsymbol{f}\|_{0, \Omega}+\left\|\boldsymbol{B}_{d}\right\|_{0, \Omega}+\left\|\operatorname{curl} \boldsymbol{B}_{d}\right\|_{\mathrm{r}, \Omega}, \\
\|\operatorname{curl} \boldsymbol{E}\|_{\mathrm{r}^{\prime}, \Omega} \lesssim\|\boldsymbol{f}\|_{0, \Omega}+\left\|\boldsymbol{B}_{d}\right\|_{\mathrm{r}^{\prime}, \Omega}+\left\|\operatorname{curl} \boldsymbol{B}_{d}\right\|_{0, \Omega} .
\end{array}\right.
$$

\section{$5 \quad H(\operatorname{curl})$-conforming finite element discretization}

Edge finite element methods are natural candidates for the numerical solution of electromagnetic problems. Since these methods lead to $\boldsymbol{H}$ (curl)-conforming approximations, some features of the numerical solutions can be rather easily studied by leveraging the results obtained for the exact problems. While the comprehensive numerical analysis of the approximate problems is out of the scope of this paper, this section aims at giving a few numerical results for the considered problems. After introducing a standard edge finite element discretization and basic results, we derive an a priori error estimate, which is obtained by using the regularity estimates. Elementary numerical results are then proposed to illustrate the expected convergence rate of the method.

\subsection{Discretization and a priori error estimate}

We consider a shape regular familly of meshes $\left(\mathcal{T}_{h}\right)_{h}$ for the domain $\Omega$. For the sake of simplicity, we assume that the domain $\Omega$ is a Lipschitz polyhedron. Each mesh $\mathcal{T}_{h}$ is made up of closed non-overlapping tetrahedra, generically denoted by $K$, and is indexed by $h:=\max _{K} h_{K}$, where $h_{K}$ is the diameter of $K$. Denoting by $\rho_{K}$ the diameter of the largest ball inscribed in $K$, we assume that there exists a shape regularity parameter $\varsigma>0$ such that for all $h$, for all $K \in \mathcal{T}_{h}$, it holds that $h_{K} \leq \varsigma \rho_{K}$.

Finite-dimensional subspaces $\left(\boldsymbol{V}_{h}\right)_{h}$ of $\boldsymbol{H}(\mathbf{c u r l} ; \Omega)$ are defined by using the so-called Nédélec's first family of edge finite elements. Elements of degree 1 are considered. One has

$$
\boldsymbol{V}_{h}:=\left\{\boldsymbol{v}_{h} \in \boldsymbol{H}(\operatorname{curl} ; \Omega), \boldsymbol{v}_{h \mid K} \in \mathcal{R}_{1}(K), \forall K \in \mathcal{T}_{h}\right\},
$$


where $\mathcal{R}_{1}(K)$ is the six-dimensional vector space of polynomials on $K$

$$
\mathcal{R}_{1}(K):=\left\{\boldsymbol{v} \in \boldsymbol{P}_{1}(K): \boldsymbol{v}(\boldsymbol{x})=\boldsymbol{a}+\boldsymbol{b} \times \boldsymbol{x}, \boldsymbol{a}, \boldsymbol{b} \in \mathbb{R}^{3}\right\} .
$$

The subspaces verify the approximability property (see e.g. Lemma 7.10 in Ref. [24])

$$
\lim _{h \rightarrow 0}\left(\inf _{\boldsymbol{v}_{h} \in \boldsymbol{V}_{h}}\left\|\boldsymbol{v}-\boldsymbol{v}_{h}\right\|_{\boldsymbol{H}(\mathbf{c u r l} ; \Omega)}\right)=0, \forall \boldsymbol{v} \in \boldsymbol{H}(\operatorname{curl} ; \Omega) .
$$

We also introduce the closed subspaces $\left(\boldsymbol{V}_{h}^{0}\right)_{h}$ with $\boldsymbol{V}_{h}^{0}:=\boldsymbol{V}_{h} \cap \boldsymbol{H}_{0}(\mathbf{c u r l} ; \Omega)$, which also verify the approximability property in $\boldsymbol{H}_{0}(\mathbf{c u r l} ; \Omega)$.

Using the standard Galerkin approach, the variational formulation of the approximate problem is obtained by seeking the solution in $\boldsymbol{V}_{h}$ with test functions in $\boldsymbol{V}_{h}^{0}$ or $\boldsymbol{V}_{h}$ for the Dirichlet and Neumann cases, respectively. Therefore, the discrete Dirichlet problem reads

$$
\left\{\begin{array}{l}
\text { Find } \boldsymbol{E}_{h} \in \boldsymbol{V}_{h} \text { such that } \\
a\left(\boldsymbol{E}_{h}, \boldsymbol{F}_{h}\right)=\ell_{\mathrm{D}}\left(\boldsymbol{F}_{h}\right), \forall \boldsymbol{F}_{h} \in \boldsymbol{V}_{h}^{0}, \\
\boldsymbol{E}_{h} \times \boldsymbol{n}=\boldsymbol{g}_{h} \text { on } \partial \Omega,
\end{array}\right.
$$

and the discrete Neumann problem reads

$$
\left\{\begin{array}{l}
\text { Find } \boldsymbol{E}_{h} \in \boldsymbol{V}_{h} \text { such that } \\
a\left(\boldsymbol{E}_{h}, \boldsymbol{F}_{h}\right)=\ell_{\mathrm{N}}\left(\boldsymbol{F}_{h}\right), \forall \boldsymbol{F}_{h} \in \boldsymbol{V}_{h},
\end{array}\right.
$$

with the sesquilinear form

$$
a:(\boldsymbol{u}, \boldsymbol{v}) \mapsto\left(\underline{\boldsymbol{\mu}}^{-1} \operatorname{curl} \boldsymbol{u} \mid \operatorname{curl} \boldsymbol{v}\right)_{0, \Omega}-\omega^{2}(\underline{\varepsilon} \boldsymbol{u} \mid \boldsymbol{v})_{0, \Omega}
$$

defined on $\boldsymbol{H}(\mathbf{c u r l} ; \Omega)$. The linear forms $\ell_{\mathrm{D}}$ and $\ell_{\mathrm{N}}$ are defined in sections 3.1 and 4.1 , respectively. The right-hand-side term $\boldsymbol{g}_{h}$ is the projection of $\boldsymbol{g}$ onto $\gamma^{T} \boldsymbol{V}_{h}$. For simplicity, in the remaining, we assume that the integrals are computed exactly.

As a first result, we derive a sharp error estimate for the interpolation of the solutions of both problems onto the finite element space. Let $\pi_{h}$ denote the classical interpolation operator from $\boldsymbol{H}_{0}(\mathbf{c u r l} ; \Omega)$ onto $\boldsymbol{V}_{h}^{0}$, resp. from $\boldsymbol{H}(\mathbf{c u r l} ; \Omega)$ onto $\boldsymbol{V}_{h}$. One has the following interpolation error estimate [7]:

Theorem 5.1. Let $\mathrm{t} \in(1 / 2,1]$ and $\mathrm{t}^{\prime} \in(0,1]$. For all $\boldsymbol{v} \in\left\{\boldsymbol{v} \in \boldsymbol{H}^{\mathrm{t}}(\Omega)\right.$, curl $\left.\boldsymbol{v} \in \boldsymbol{H}^{\mathrm{t}^{\prime}}(\Omega)\right\}$, it holds that

$$
\left\|\boldsymbol{v}-\pi_{h} \boldsymbol{v}\right\|_{\boldsymbol{H}(\operatorname{curl} ; \Omega)} \lesssim h^{\min \left(\mathrm{t}, \mathrm{t}^{\prime}\right)}\left(\|\boldsymbol{v}\|_{\mathrm{t}, \Omega}+\|\operatorname{curl} \boldsymbol{v}\|_{\mathrm{t}^{\prime}, \Omega}\right) .
$$

In this result, $t>1 / 2$ is assumed for simplicity. A similar result can be obtained for $t \in(0,1]$ with the help of the combined interpolation operator (see Section 4.2 in Ref. [10]), but this result is more involved. Indeed, the norm of the gradient part of the decomposition of $\boldsymbol{v}$ (given in theorems 3.2 or 3.5) then appears in the right-hand side of (59), in addition to both terms already there. Nevertheless, since the gradient part is bounded by the norm of the data (see again theorems 3.2 or 3.5), the same conclusion stands in this general case. Then, observe that one can replace the field $\boldsymbol{v}$ with $\boldsymbol{E}$ in (59). Using theorems 3.8 and 4.4, the norms $\|\boldsymbol{E}\|_{\mathrm{t}, \Omega}$ and $\|\operatorname{curl} \boldsymbol{E}\|_{\mathrm{t}^{\prime}, \Omega}$ are bounded by the norms on the data, and the exponents become $t=\min (\mathbf{s}, r)$ and $t^{\prime}=r^{\prime}$, where $s, r, r^{\prime}$ are the extra-regularity exponents for the data. Injecting the regularity estimates in (59) then gives

$$
\left\|\boldsymbol{E}-\pi_{h} \boldsymbol{E}\right\|_{\boldsymbol{H}\left(\mathbf{c u r l} ; \Omega_{h}\right)} \lesssim h^{\min \left(\mathbf{s}, \mathbf{r}, \mathbf{r}^{\prime}\right)},
$$


where the bounds on the exponents are defined in theorems 3.8 and 4.4 for the Dirichlet and Neumann cases, respectively.

In order to derive an a priori error estimate for both problems, one has to bound the error between the numerical solution and the exact solution with the interpolation error. For a problem with a coercive sesquilinear form, it is known that an a priori error estimate for the numerical solution is obtained thanks to Céa's lemma.

Theorem 5.2. When the sesquilinear form $a(\cdot, \cdot)$ is coercive, it holds that

$$
\exists C>0, \forall h,\left\|\boldsymbol{E}-\boldsymbol{E}_{h}\right\|_{\boldsymbol{H}(\operatorname{curl} ; \Omega)} \leq C \inf _{\boldsymbol{w}_{h} \in \boldsymbol{V}_{h}}\left\|\boldsymbol{E}-\boldsymbol{w}_{h}\right\|_{\boldsymbol{H}(\mathbf{c u r l} ; \Omega)} .
$$

Using $\boldsymbol{w}_{h}=\pi_{h} \boldsymbol{E}$ and the estimates (60) and (61), one has that

$$
\left\|\boldsymbol{E}-\boldsymbol{E}_{h}\right\|_{\boldsymbol{H}(\mathbf{c u r l} ; \Omega)} \lesssim h^{\min \left(\mathbf{s}, \mathbf{r}, \mathbf{r}^{\prime}\right)},
$$

where the exponents depend only on the regularity of the data.

Let us highlight that the regularity results have been obtained for a boundary of class $\mathcal{C}^{2}$, while the interpolation error estimates are for Lipschitz polyhedral domains. The error resulting from this geometric approximation can be studied thanks to the framework introduced by Dello Russo and Alonso [15]. Following Section 8 there, one obtains additional terms in the right-hand side of (61), which are asymptotically all in the order of $O(h)$.

On the other hand, to obtain a similar estimate for a problem with a non-coercive sesquilinear form, one has to prove a uniform discrete inf-sup condition and to combine it with a generalised Céa's lemma. In our case, when $a(\cdot, \cdot)$ is not coercive, deriving a uniform discrete inf-sup condition requires tedious developments. We refer the reader to [18] and [11] for analyses in slightly different contexts. Provided that such a result is available, the estimate (62) holds.

\subsection{Numerical results with a manufactured benchmark}

To illustrate the expected convergence rate with a numerical case, we consider a simple benchmark with a manufactured solution. Let a spherical domain of unit radius centered at the origin, $\Omega=\left\{\boldsymbol{x} \in \mathbb{R}^{3},\|\boldsymbol{x}\|<1\right\}$, the angular frequency $\omega=1$ and the material tensors

$$
\underline{\boldsymbol{\mu}}=\operatorname{diag}(1,1,1), \quad \underline{\boldsymbol{\varepsilon}}=\operatorname{diag}\left(1+10^{-1} i, 1+10^{-1} i,-2+10^{-1} i\right),
$$

which fulfill assumption (2). According to Lemma 2.3 in Ref. [25], the sesquilinear form $a$ is coercive for these material tensors. A nonhomogeneous Dirichlet or Neumann boundary condition is prescribed on the boundary of the domain. We consider the manufactured solution

$$
\boldsymbol{E}_{\mathrm{ref}}=[-1,1,1]^{\top} \exp (i \pi \boldsymbol{k} \cdot \boldsymbol{x}), \quad \text { with } \boldsymbol{k}=\frac{1}{\sqrt{14}}[3,2,1]^{\top} .
$$

The volume source term is chosen accordingly, i.e. $\boldsymbol{f}=\operatorname{curl} \operatorname{curl} \boldsymbol{E}_{\mathrm{ref}}-\omega^{2} \underline{\boldsymbol{\varepsilon}} \boldsymbol{E}_{\mathrm{ref}}$, as well as the right-hand-side term of the boundary conditions.

Numerical simulations are performed with FreeFem [20] using six unstructured meshes made of tetrahedra and first-degree edge finite elements. Because the boundary of the meshes (which are polyhedral) does not exactly match the curved border of the spherical domain, the boundary data used in the numerical simulation are evaluated on the sphere and then projected on the surface mesh. It has been proven that this geometric approximation introduces a geometric error of the order $\mathcal{O}(h)[15]$. 


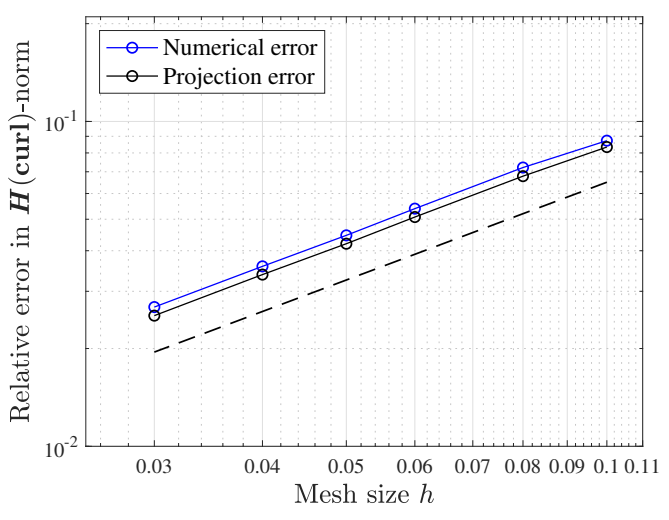

(a) Dirichlet case

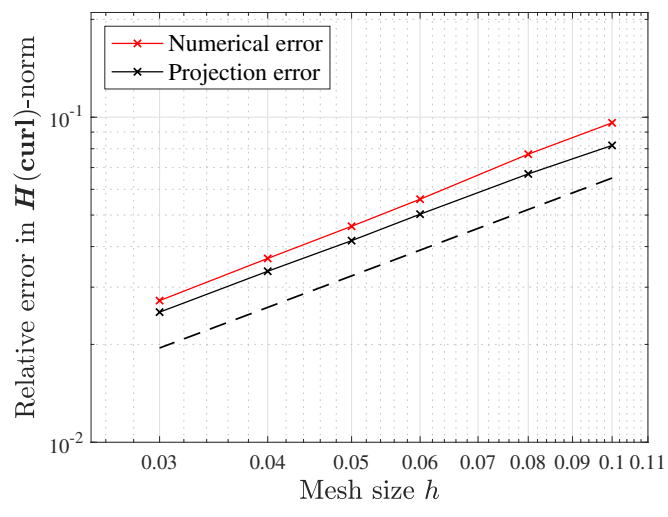

(b) Neumann case

Figure 1: Relative error in $\boldsymbol{H}$ (curl)-norm obtained with six meshes for the manufactured benchmark with Dirichlet and Neumann boundary conditions. The relative projection error in $\boldsymbol{H}(\mathbf{c u r l})$-norm is plotted in both cases. The dashed lines correspond to the linear scale $O(h)$.

The relative numerical error in $\boldsymbol{H}$ (curl)-norm is plotted as a function of the mesh size $h$ in Fig. 1 for both Dirichlet and Neumann cases. As a reference, the relative error corresponding to the projection of the reference solution on the discrete solution space, which corresponds to the best approximation error according to Céa's lemma, is plotted as well. As the solution $\boldsymbol{E}_{\text {ref }}$ is smooth, it belongs to $\boldsymbol{H}^{1}(\Omega)$ as well as its curl, and one has $\mathrm{t}=\mathrm{t}^{\prime}=1 \mathrm{in}$ (62). Therefore, one expects the error to evolve linearly with the mesh size $h$. The results reported in Fig. 1 show that the convergence behaves effectively like $O(h)$ for both problems.

\section{Conclusion and extensions}

We have addressed the mathematical analysis of time-harmonic electromagnetic boundary value problems in complex anisotropic material tensors, which fulfill a general ellipticity condition. After having developed a functional framework suited to these complex material tensors (i.e. function spaces, Helmholtz decompositions, Weber inequalities, and compact embedding results), we have analyzed the well-posedness of the Dirichlet and Neumann problems (see Theorems 3.1 and 4.1, respectively), as well as the regularity of the solution (i.e. the electric field) and the solution's curl. The regularity results are summarized in Theorems 3.8 and 4.4. A preliminary numerical analysis with a $\boldsymbol{H}(\mathbf{c u r l})$-conforming finite element discretization has been proposed.

Among possible extensions, one could consider a domain $\Omega$ with a non-smooth boundary (e.g. polyhedral), possibly non-convex. The shift theorems then have to be revisited. To our knowledge, results are available for piecewise smooth, Hermitian and elliptic material tensors (see e.g. $[13,19,8,12]$ for shift theorems in settings with a non-smooth boundary and Hermitian tensors). The theory developed in this work could be extended thanks to these results. One could also consider settings with mixed boundary conditions, i.e. when a Dirichlet condition is prescribed on one part of the boundary, and a Neumann condition is prescribed on the rest of the boundary. To carry out the theory and fit the problem within the coercive+compact framework, we refer the reader to [16]. For shift theorems, we refer the reader to the works of Jochmann [22]. Finally, one could consider variational formulations with the magnetic field as the unknown, which poses no extra difficulty. 


\section{A Additional definitions}

Definition A.1. From a topological point of view, a domain $\Omega$ verifies the hypothesis (Top) $)_{I}$ if one of the following conditions holds:

1. For all curl-free vector field $\boldsymbol{v} \in C^{1}(\Omega)$, there exists $p \in C^{0}(\Omega)$ such that $\boldsymbol{v}=\nabla p$ in $\Omega$.

2. There exist $I>0$ non-intersecting, piecewise plane manifolds, $\left(\Sigma_{j}\right)_{j=1, \ldots, I}$, with boundaries $\partial \Sigma_{i} \subset \partial \Omega$, such that, if we let $\dot{\Omega}=\Omega \backslash \bigcup_{i=1}^{I} \Sigma_{i}$, for all curl-free vector field $\boldsymbol{v}$, there exists $\dot{p} \in C^{0}(\dot{\Omega})$ such that $\boldsymbol{v}=\nabla \dot{p}$ in $\dot{\Omega}$.

If the first condition holds, $\Omega$ is topologically trivial, and we set $I=0$ and $\dot{\Omega}=\Omega$. If the second condition holds, $\Omega$ is topologically non-trivial. See [17] for further details.

The set $\dot{\Omega}$ has pseudo-Lipschitz boundary in the sense of [4]. The extension operator from $L^{2}(\dot{\Omega})$ to $L^{2}(\Omega)$ is denoted by ${ }^{\sim}$, whereas the jump across $\Sigma_{i}$ is denoted by $[\cdot]_{\Sigma_{i}}$, for $i=1, \cdots, I$. The definition of the jump depends on the (fixed) orientation of the normal vector field to $\Sigma_{i}$. One has the integration by parts formula [4]:

$$
(\boldsymbol{v} \mid \nabla \dot{q})_{0, \dot{\Omega}}+(\operatorname{div} \boldsymbol{v} \mid \dot{q})_{0, \dot{\Omega}}=\sum_{1 \leq j \leq I}\left\langle\boldsymbol{v} \cdot \boldsymbol{n},[\dot{q}]_{\Sigma_{j}}\right\rangle_{H^{1 / 2}\left(\Sigma_{j}\right)}, \forall \boldsymbol{v} \in \boldsymbol{H}_{0}(\operatorname{div} ; \Omega), \forall \dot{q} \in H^{1}(\dot{\Omega}) .
$$

For the sake of completeness, we recall Definition 3.6.3 of Ref. [5].

Definition A.2. A domain $\Omega$ is said of the $\mathfrak{A}$-type if, for any $\boldsymbol{x} \in \partial \Omega$, there exists a neighbourhood $\mathcal{V}$ of $\boldsymbol{x}$ in $\mathbb{R}^{3}$, and a $\mathcal{C}^{2}$ diffeomorphism that transforms $\Omega \cap \mathcal{V}$ into one of the following types, where $\left(x_{1}, x_{2}, x_{3}\right)$ denote the Cartesian coordinates and $(\rho, \omega) \in \mathbb{R} \times \mathbb{S}^{2}$ the spherical coordinates in $\mathbb{R}^{3}$ :

1. $\left[x_{1}>0\right]$, i.e. $\boldsymbol{x}$ is a regular point;

2. $\left[x_{1}>0, x_{2}>0\right]$, i.e. $\boldsymbol{x}$ is a point on a salient (outward) edge;

3. $\mathbb{R}^{3} \backslash\left[x_{1} \geq 0, x_{2} \geq 0\right]$, i.e. $\boldsymbol{x}$ is a point on a reentrant (inward) edge;

4. $[\rho>0, \omega \in \tilde{\Omega}]$, where $\tilde{\Omega} \subset \mathbb{S}^{2}$ is a topologically trivial domain. In particular, if $\partial \tilde{\Omega}$ is smooth, $\boldsymbol{x}$ is a conical vertex; if $\partial \tilde{\Omega}$ is a made of arcs of great circles, $\boldsymbol{x}$ is a polyhedral vertex.

\section{Acknowledgment}

The authors thank DGA (Direction Générale de l'Armement) for the doctoral scholarship of the first author.

\section{References}

[1] G. S. Alberti. Hölder regularity for Maxwell's equations under minimal assumptions on the coefficients. Calculus of Variations and Partial Differential Equations, 57(3):71, 2018.

[2] G. S. Alberti and Y. Capdeboscq. Elliptic regularity theory applied to time harmonic anisotropic Maxwell's equations with less than Lipschitz complex coefficients. SIAM Journal on Mathematical Analysis, 46(1):998-1016, 2014.

[3] A. Alonso and A. Valli. Unique solvability for high-frequency heterogeneous time-harmonic Maxwell equations via the Fredholm alternative theory. Mathematical Methods in the Applied Sciences, 21(6):463-477, 1998.

[4] C. Amrouche, C. Bernardi, M. Dauge, and V. Girault. Vector potentials in three-dimensional non-smooth domains. Mathematical Methods in the Applied Sciences, 21:823-864, 1998. 
[5] F. Assous, P. Ciarlet Jr., and S. Labrunie. Mathematical foundations of computational electromagnetism, volume 198 of Applied Mathematical Sciences. Springer, 2018.

[6] A. Back, T. Hattori, S. Labrunie, J.R. Roche, and P. Bertrand. Electromagnetic wave propagation and absorption in magnetised plasmas: variational formulations and domain decomposition. Math. Model. Numer. Anal., 49:1239-1260, 2015.

[7] A. Bermúdez, R. Rodríguez, and P. Salgado. Numerical treatment of realistic boundary conditions for the eddy current problem in an electrode via Lagrange multipliers. Mathematics of computation, 74(249):123-151, 2005.

[8] A. Bonito, J.-L. Guermond, and F. Luddens. Regularity of the Maxwell equations in heterogeneous media and Lipschitz domains. Journal of Mathematical Analysis and Applications, 408:498-512, 2013.

[9] A. Buffa, M. Costabel, and D. Sheen. On traces for $\boldsymbol{H}(\mathbf{c u r l} ; \Omega)$ in Lipschitz domains. Journal of Mathematical Analysis and Applications, 276:845-867, 2002.

[10] P. Ciarlet, Jr. On the approximation of electromagnetic fields by edge finite elements. Part 1: Sharp interpolation results for low-regularity fields. Computers 8 Mathematics with Applications, 71:85-104, 2016.

[11] P. Ciarlet, Jr. Mathematical and numerical analyses for the div-curl and div-curlcurl problems with a sign-changing coefficient. https://hal.archives-ouvertes.fr/hal-02567484, May 2020.

[12] P. Ciarlet, Jr. On the approximation of electromagnetic fields by edge finite elements. Part 3: Sensitivity to coefficients. SIAM Journal on Mathematical Analysis, 52(3):3004-3038, 2020.

[13] M. Costabel, M. Dauge, and S. Nicaise. Singularities of Maxwell interface problems. ESAIM: Mathematical Modelling and Numerical Analysis, 33:627-649, 1999.

[14] M. Costabel, M. Dauge, and S. Nicaise. Corner singularities and analytic regularity for linear elliptic systems. Part I: Smooth domains. https://hal .archives-ouvertes.fr/hal-00453934, February 2010.

[15] A. Dello Russo and A. Alonso. Finite element approximation of Maxwell eigenproblems on curved Lipschitz polyhedral domains. Applied numerical mathematics, 59(8):1796-1822, 2009.

[16] P. Fernandes and G. Gilardi. Magnetostatic and electrostatic problems in inhomogeneous anisotropic media with irregular boundary and mixed boundary conditions. Mathematical Models and Methods in Applied Sciences, 7:957-991, 1997.

[17] P.W. Gross and P.R. Kotiuga. Electromagnetic theory and computation: a topological approach. MSRI Publications Series, 48. Cambridge University Press, 2004.

[18] M. Halla. Electromagnetic Steklov eigenvalues: approximation analysis. ESAIM: M2AN, 55(1):5776, 2021.

[19] R. Haller-Dintelmann, H.-C. Kaiser, and J. Rehberg. Direct computation of elliptic singularities across anisotropic, multi-material edges. Journal of Mathematical Sciences, 172:589-622, 2011.

[20] F. Hecht. New development in FreeFem++. Journal of Numerical Mathematics, 20:251-265, 2012.

[21] R. Hiptmair. Finite elements in computational electromagnetics. Acta Numerica, pages 237-339, 2002.

[22] Frank Jochmann. Regularity of weak solutions of Maxwell's equations with mixed boundaryconditions. Mathematical methods in the applied sciences, 22(14):1255-1274, 1999.

[23] A. Kirsch and F. Hettlich. Mathematical Theory of Time-harmonic Maxwell's Equations. Springer, 2016.

[24] P. Monk. Finite element methods for Maxwell's equations. Oxford University Press, 2003.

[25] E. Sébelin, Y. Peysson, X. Litaudon, D. Moreau, J.C. Miellou, and O. Lafitte. Uniqueness and existence result around Lax-Milgram lemma: application to electromagnetic waves propagation in tokamak plasmas. Technical report, Association Euratom-CEA, 1997. http://www.iaea.org/ inis/collection/NCLCollectionStore/_Public/30/017/30017036.pdf.

[26] B. Tsering-xiao and W. Xiang. Regularity of solutions to time-harmonic Maxwell's system with various lower than Lipschitz coefficients. Nonlinear Analysis, 192:111693, 2020.

[27] X. Xiang. On $L^{r}$ estimates for Maxwell's equations with complex coefficients in Lipschitz domains. SIAM Journal on Mathematical Analysis, 52(6):6140-6154, 2020. 